\title{
A new approach to the $N$-particle problem in QM
}

\author{
JOACHIM SCHRÖTER
}

In this paper the old problem of determining the discrete spectrum of a multi-particle Hamiltonian is reconsidered. The aim is to bring a fermionic Hamiltonian for arbitrary numbers $N$ of particles by analytical means into a shape such that modern numerical methods can successfully be applied. For this purpose the Cook-Schroeck Formalism is taken as starting point. This includes the use of the occupation number representation. It is shown that the $N$-particle Hamiltonian is determined in a canonical way by a fictional 2particle Hamiltonian. A special approximation of this 2-particle operator delivers an approximation of the $N$-particle Hamiltonian, which is the orthogonal sum of finite dimensional operators. A complete classification of the matrices of these operators is given. Finally the method presented here is formulated as a work program for practical applications. The connection with other methods for solving the same problem is discussed.

\section{Introduction}

One of the central problems of many-particle quantum mechanics, if not its main problem, is calculating the spectral representation of a many-particle Hamiltonian, which typically has the form

$$
\mathbf{H}_{N}=\sum_{j}^{N} K_{j}+\frac{1}{2} \sum_{j \neq k}^{N} W_{j k} .
$$

Here $K_{j}$ contains the kinetic energy of particle $j$ and the external fields acting upon $j$, and $W_{j k}$ is the interaction of the particles $j$ and $k$. As is well-known, this problem has a solution if $W_{j k}=0$. On the other hand, if $W_{j k}$ does not vanish, the problem is "almost" unsolvable in a strict sense. But the situation is not hopeless. For, what is really needed for practical purposes, is a "good" approximate solution. 
In this last field a tremendous work has been done, both analytically and numerically. Its mainstreams are well-known under the labels Thomas-Fermi method ([1],[2]), Hartree-Fock method ([3],[4]), density functional theory $([5],[6])$, configuration interaction method, Haken's method and others. With respect to these methods and their applications and refinements I refer e.g. to the following books [7], [8], [9]. There in addition an abundance of papers and monographs is cited, where the methods are also described in detail.

A common feature of these procedures is that they contain one step in which a one-particle approximation of the $N$-particle problem is carried through. With the methods of Thomas-Fermi and of Hartree-Fock it is all, what is done. With the other methods the described first step is followed by other ones thereby improving the accuracy of approximation. Especially, by combining analytical and numerical mathematics great progress is achieved. Today problems can be solved which were regarded as unsolvable few decades ago.

Nevertheless, the question is obvious, whether there are other approaches to a solution of the $N$-particle problem in quantum mechanics than those mentioned above. It is the aim of this paper to present such a new procedure. For this purpose I need some mathematical tools which, though they are widely known, I have briefly described in Appendix A.1. In particular the reader will find all the notation which is used throughout the text. (More details can be found in [10], [11].) The basic idea of the procedure as well as the main results are sketched in Section 2.3.

\section{The Structure of $N$-Particle Hamiltonians}

2.1: In what follows only systems of particles of the same kind are considered. When one starts studying a concrete sytem, its Hamiltonian is usually defined using the position-spin representation, i.e. the Hamiltonian is an operator in the Hilbert space $\bigotimes^{N}\left(L^{2}\left(\mathbb{R}^{3}\right) \otimes \mathcal{S}^{1}\right)$, where $\otimes$ and $\otimes$ denote tensor products, and where $\mathcal{S}^{1}$ is the complex vector space of spin functions (cf. Section A.2.1). For explicit calculations this representation is very useful. But the aim of this paper is primarily a structural analysis of the Hamiltonians of a certain class of systems, and in this case a more abstract formalism is adequate. It turns out that the Cook-Schroeck formalism (cf. Appendix A.1) is very useful for this purpose.

Then our starting point is an arbitrary initial Hamiltonian of the shape (1.1), which is denoted $\bar{H}_{N}$ and defined in a Hilbert space $\overline{\mathcal{H}}^{N}:=$ $\bigotimes^{N} \overline{\mathcal{H}}^{1}$, where $\overline{\mathcal{H}}^{1}$ is the Hilbert space of the corresponding one-particle system. 
Now let $K$ be the operator defined in $\overline{\mathcal{H}}^{1}$ which contains the kinetic energy of one particle of a certain kind together with the action of the external fields. Moreover, let $W$ be that operator in $\overline{\mathcal{H}}^{2}$ which represents the interaction of two particles of the kind considered. Then, using Formula (A.1.25), $\bar{H}_{N}$ defined in $\overline{\mathcal{H}}^{N}$ is given by

$$
\bar{H}_{N}=\Omega_{N}(K)+\Omega_{N}(W),
$$

where

$$
\begin{aligned}
& \Omega_{N}(K):=((N-1) !)^{-1} \sum_{P \in \mathcal{S}_{N}} U(P)(K \otimes 1 \otimes \cdots \otimes 1) U^{\star}(P), \\
& \Omega_{N}(W):=(2(N-2) !)^{-1} \sum_{P \in \mathcal{S}_{N}} U(P)(W \otimes 1 \otimes \cdots \otimes 1) U^{\star}(P),
\end{aligned}
$$

and $U(P)$ is the unitary permutation operator defined by the particle permutation P. Thus, using Formula (A.1.27), the operator $\bar{H}_{N}$ specified for Bosons or Fermions reads

$$
\bar{H}_{N}^{ \pm}=\Omega_{N}^{ \pm}(K)+\Omega_{N}^{ \pm}(W) .
$$

Here the definition $A^{ \pm}:=S_{N}^{ \pm} A S_{N}^{ \pm}$for an arbitrary operator $A$ in $\overline{\mathcal{H}}^{N}$ is applied, where $S_{N}^{ \pm}$is the symmetrizer (+) resp. the antisymmetrizer (-). Then $A^{ \pm}$is defined in the Hilbert space $\overline{\mathcal{H}}_{ \pm}^{N}=S_{N}^{ \pm}\left[\overline{\mathcal{H}}^{N}\right]$.

It is well-known that the structure of $\bar{H}_{N}^{ \pm}$given by (2.3) is not helpful for studying its spectral problem, because the operators $\Omega_{N}^{ \pm}(K)$ and $\Omega_{N}^{ \pm}(W)$ do not commute. This suggests the question if it is possible to find an operator $T$ acting in $\overline{\mathcal{H}}^{M}, 1 \leq M<N$ such that

$$
\bar{H}_{N}^{ \pm}=\Omega_{N}^{ \pm}(T) .
$$

Because the two-particle operator $W$ cannot be represented by a one-particle operator it holds that $M \geq 2$. If the Hamiltonians as well as the operators $K$ and $W$ are selfadjoint, it turns out that $M=2$ is possible as shown by the following

Proposition 2.1. Let

$$
\tilde{H}_{2}(\gamma)=\gamma(K \otimes 1)+\gamma(1 \otimes K)+W
$$


so that $\tilde{H}_{2}(\gamma)$ is defined in $\overline{\mathcal{H}}^{2}$, and let $\gamma_{0}:=(N-1)^{-1}$. Then

$$
\bar{H}_{N}^{ \pm}=\Omega_{N}^{ \pm}\left(\tilde{H}_{2}\left(\gamma_{0}\right)\right) \quad \text { and } \quad \bar{H}_{N}^{ \pm} \neq \Omega_{N}^{ \pm}\left(\tilde{H}_{2}(\gamma)\right), \quad \gamma \neq \gamma_{0} .
$$

Proof. Using (A.1.28) yields

$$
\begin{aligned}
\Omega_{N}^{ \pm}(K)= & N S_{N}^{ \pm}(K \otimes 1 \otimes \cdots \otimes 1) S_{N}^{ \pm} \\
= & \frac{1}{N-1}\left(\begin{array}{c}
N \\
2
\end{array}\right) S_{N}^{ \pm}((K \otimes 1) \otimes \cdots \otimes 1) S_{N}^{ \pm} \\
& +\frac{1}{N-1}\left(\begin{array}{c}
N \\
2
\end{array}\right) S_{N}^{ \pm}((1 \otimes K) \otimes \cdots \otimes 1) S_{N}^{ \pm} \\
= & \frac{1}{N-1}\left(\Omega_{N}^{ \pm}(K \otimes 1)+\Omega_{N}^{ \pm}(1 \otimes K)\right) .
\end{aligned}
$$

Since by supposition $\tilde{H}_{2}(\gamma), K$ and $W$ are selfadjoint, (A.1.30) can be applied so that with the help of (2.7) the following relation holds:

$$
\begin{aligned}
\Omega_{N}^{ \pm}\left(\tilde{H}_{2}(\gamma)\right) & \supset \gamma \Omega_{N}^{ \pm}(K \otimes 1)+\gamma \Omega_{N}^{ \pm}(1 \otimes K)+\Omega_{N}^{ \pm}(W) \\
& =\gamma(N-1) \Omega_{N}^{ \pm}(K)+\Omega_{N}^{ \pm}(W) .
\end{aligned}
$$

The term in the last line of (2.8) is selfadjoint because it is the Hamiltonian of a (possibly fictional) $N$-particle system. Since also $\Omega_{N}^{ \pm}\left(\tilde{H}_{2}(\gamma)\right)$ is selfadjoint (cf. Proposition A.1.11), relation (2.8) is an equation, from which the proposition follows immediately.

2.2: This result is somewhat surprising. The initial Hamiltonian $\bar{H}_{N}^{ \pm}$is not determined by $\tilde{H}_{2}(1)$, i.e. by a Hamiltonian of a system of two particles of the same kind, which is described by $\bar{H}_{N}^{ \pm}$. Rather $\bar{H}_{N}^{ \pm}$is determined by $\tilde{H}_{2}\left(\gamma_{0}\right), \gamma_{0}^{-1}=N-1$, which is a two-particle Hamiltonian for particles of mass $(N-1) m_{0}$ and external fields weakened by a factor $(N-1)^{-1}$, but with the same interaction $W$ as the particles described by $\bar{H}_{N}^{ \pm}$, which are supposed to have mass $m_{0}$.

The system described by $\tilde{H}_{2}\left(\gamma_{0}\right)$ is fictional. I call it dummy system and the operator $\tilde{H}_{2}\left(\gamma_{0}\right)$ dummy Hamiltonian.

In Appendix A.2, two simple examples are given describing dummy helium and a solid with two dummy electrons.

In what follows, the operator $\tilde{H}_{2}(\gamma), \gamma \neq \gamma_{0}$ is not needed anymore. Therefore it is convenient to use the notation $\tilde{H}_{2}\left(\gamma_{0}\right)=\bar{H}_{20}$.

Corollary 2.2. Because of (A.1.31) it follows that

$$
\bar{H}_{N}^{ \pm}=\Omega_{N}^{ \pm}\left(\bar{H}_{20}\right)=\Omega_{N}^{ \pm}\left(\bar{H}_{20}^{ \pm}\right) .
$$


2.3: Formula (2.9) suggests the basic idea of this paper: find an approximation of $\bar{H}_{N}^{ \pm}$via an approximation of $\bar{H}_{20}^{ \pm}$such that the spectral problem of $\bar{H}_{N}^{ \pm}$can be solved approximately.

The details of this program are carried out for fermions in four steps, which correspond to the Sections 3 to 6 .

Section 3 contains a formal analysis of a restriction $H_{N}^{-}$of the initial Hamiltonian $\bar{H}_{N}^{-}$. The aim is expressing the matrix elements of $H_{N}^{-}$in terms of the matrix elements of the restricted dummy operator $H_{20}^{-}$, which is bounded. The results are summarized in Proposition 3.10.

In order to use them for the present purposes, a properly chosen orthonormal system $\mathcal{O}_{1}$ in the one-particle Hilbert space $\overline{\mathcal{H}}^{1}$ is needed. In Section 4 arguments are given that such a system is obtained via the Hartree-Fock procedure applied to $\bar{H}_{20}^{-}$. Thus the restrictions used in Section 3 can be justified. Moreover, a heuristic argumentation suggests that $H_{20}^{-}$can be "truncated" such that an operator $\hat{H}_{20}^{-}$results, which is, depending on a parameter $\alpha \in \mathbb{N}$, an approximation of $H_{20}^{-}$.

In Section 5 it is shown that the operators $\hat{H}_{20}^{-}$converge strongly to $H_{20}^{-}$, if $\alpha \rightarrow \infty$. This has the consequence that the operators $\hat{H}_{N}^{-}=\Omega_{N}^{-}\left(\hat{H}_{20}^{-}\right)$also converge strongly to $H_{N}^{-}$. Therefore it is possible to apply the results of the theory of spectral approximation (cf. e.g. [12],[13]).

Finally, in Section 6 an analysis of the operators $\hat{H}_{N}^{-}$is given. It is shown that they are block-diagonal, i.e. their matrices with respect to the chosen orthogonal basis are orthogonal sums of finite dimensional matrices, the structure of which are analyzed in detail. At this point numerical methods can come into play.

In Section 7 the results of the previous sections are summarized in the form of a work program, which can be regarded as the main result of this paper.

\section{The Hamiltonian $H_{N}^{-}$and its Matrix}

\subsection{Preliminary remarks}

3.1.1: Since in what follows only such systems are considered, which consist of fermions of the same kind, the notation introduced in Appendix A.1 can be used throughout. The Hamiltonians of these systems usually have the following property.

1.) They are unbounded, but bounded from below. 
2.) Their spectrum below a certain value $\epsilon_{0}$ is discrete, otherwise continuous with possibly inserted discrete values.

Since in this paper we are only interested in the discrete spectrum outside the continuum, i.e. in the bound states of the system, the question is obvious, whether it is possible to restrict the spectral problem to the discrete eigenvalues. In other words: is it possible to realize the following

Assumption 3.1. There is a subspace $\mathcal{H}^{1} \subset \overline{\mathcal{H}}^{1}$ such that the restriction $H_{20}^{-}$of $\bar{H}_{20}^{-}$to the subspace $\mathcal{H}_{-}^{2} \subset \overline{\mathcal{H}}_{-}^{2}$ is bounded (so that it can be defined on $\mathcal{H}_{-}^{2}$ ), and has the same discrete eigenvalues as $\bar{H}_{20}^{-}$outside its continuous spectrum.

In this section it is assumed that such a subspace $\mathcal{H}^{1} \subset \overline{\mathcal{H}}^{1}$ exists. Then $H_{N}^{-}=\Omega_{N}^{-}\left(H_{20}^{-}\right)$is bounded and defined on $\mathcal{H}_{-}^{N}$. This operator is the subject studied in the following sections. In Section 4 arguments are given that the assumption can be realized.

3.1.2: The starting point for the further considerations is the following

Notation 3.2. 1.) Let $\mathcal{B}_{1}=\left\{\phi_{\kappa}: \kappa \in \mathbb{N}\right\}$ be an arbitrary ONB in $\mathcal{H}^{1}$, and let

$$
\phi_{\kappa_{1} \cdots \kappa_{M}}:=\phi_{\kappa_{1}} \otimes \cdots \otimes \phi_{\kappa_{M}} \in \mathcal{H}^{M}
$$

with $2 \leq M \leq N$ and $\kappa_{j} \in \mathbb{N}, j=1, \ldots, M$. Then an ONB $\mathcal{B}_{M}^{-} \subset \mathcal{H}_{-}^{M}$ is defined by the vectors

$$
\Psi_{\kappa_{1} \cdots \kappa_{M}}^{-}:=\sqrt{M !} S_{M}^{-} \phi_{\kappa_{1} \cdots \kappa_{M}}
$$

with $S_{M}^{-}$being the antisymmetrizer (cf. (A.1.6)).

2.) For each sequence $\kappa_{1} \cdots \kappa_{M}$ of indices there is an infinite sequence $\hat{k}:=\left(k_{1}, k_{2}, k_{3}, \ldots,\right)$ of so called ocupation numbers $k_{\kappa}$ defined by

$$
k_{\kappa}=\sum_{j=1}^{M} \delta_{\kappa \kappa_{j}} .
$$

Hence $k_{\kappa}=1$ or 0 . Moreover there is a one-to-one correspondence

$$
\hat{k} \longleftrightarrow \kappa_{1} \cdots \kappa_{M}
$$


Thus we can write

$$
\Psi_{\kappa_{1} \cdots \kappa_{M}}^{ \pm}=: \Psi_{M}^{ \pm}(\hat{k})
$$

3.) The term "sequence of occupation numbers" is abbreviated by $b z f$ and the set of all $b z f$, which have exactly $M$ numbers 1 is denoted $B Z F_{M}$. The set $B Z F$ comprises all $b z f$.

Now let $\langle\cdot, \cdot\rangle_{2}$ be the inner product in $\mathcal{H}_{-}^{2}$ and let

$$
E(\hat{k}, \hat{m}):=\left\langle\Psi_{2}^{-}(\hat{k}), H_{20}^{-} \Psi_{2}^{-}(\hat{m})\right\rangle_{2}
$$

be the matrix elements of the dummy Hamiltonian. Then the matrix representation of $H_{20}^{-}$reads:

$$
H_{20}^{-}=\sum_{\hat{k}} \sum_{\hat{m}} E(\hat{k}, \hat{m}) \Psi_{2}^{-}(\hat{k})\left\langle\Psi_{2}^{-}(\hat{m}), \cdot\right\rangle_{2} .
$$

Using the abbreviation

$$
\Psi_{2}^{-}(\hat{k})\left\langle\Psi_{2}^{-}(\hat{m}), \cdot\right\rangle_{2}=: T(\hat{k}, \hat{m})
$$

together with Formula (A.1.28) yields:

$$
\begin{aligned}
H_{N}^{-} & =\left(\begin{array}{c}
N \\
2
\end{array}\right) S_{N}^{-}\left(H_{20}^{-} \otimes 1 \otimes \cdots \otimes 1\right) S_{N}^{-} \\
& =\left(\begin{array}{c}
N \\
2
\end{array}\right) \sum_{\hat{k}} \sum_{\hat{m}} E(\hat{k}, \hat{m}) S_{N}^{-}(T(\hat{k}, \hat{m}) \otimes 1 \otimes \cdots \otimes 1) S_{N}^{-}
\end{aligned}
$$

Remark 3.3. Here and in what follows the sums $\sum_{\hat{k}}$ and $\sum_{\hat{m}}$ are understood to run over all $b z f$, which occur in the elements of $\mathcal{B}_{2}^{-}$. Each of these sums can be arbitrarily ordered because each ordering of the $\hat{k}$ or the $\hat{m}$ yields an ONB. Since $H_{20}^{-}$is assumed to be bounded the sums $\sum_{\hat{k}}$ and $\sum_{\hat{m}}$ can be interchanged.

Notation 3.4. 1.) As usual the abbreviation

$$
\left\langle\hat{n}^{\prime}\left|H_{N}^{-}\right| \hat{n}\right\rangle:=\left\langle\Psi_{N}^{-}\left(\hat{n}^{\prime}\right), H_{N}^{-} \Psi_{N}^{-}(\hat{n})\right\rangle
$$

is used.

2.) Let $\hat{n} \in B Z F_{j}$. In the present case $j=2$ or $j=N$, and in the next section also $j=M$ is used with $2 \leq M<N$. But irrespective of these special 
choices, for each two bzf an addition and a subtraction can be defined by adding, respectively by subtracting their components. Since these operations on two $b z f$ not necessarily result in a $b z f$ the following notation is used (cf. section(A.1.3)): " $\hat{n} \pm \hat{m}$ is a $b z f$ " or " $\hat{n} \pm \hat{m} \in B Z F "$. These expressions indicate that the sequence $\hat{n} \pm \hat{m}$ does not contain the numbers 2 or -1 .

\subsection{The basic lemma}

3.2.1: It will be shown that the following proposition holds.

Lemma 3.5. There is a function $C$ such that for each triple $(\hat{n}, \hat{k}, \hat{m})$ of bzf with $\sum_{\alpha} n_{\alpha}=N, \sum_{\beta} k_{\beta}=\sum_{\beta} m_{\beta}=2$ the relations

$$
C(\hat{n}, \hat{k}, \hat{m})= \begin{cases} \pm 1, & \text { if } \hat{n}-\hat{m} \in B Z F \text { and } \hat{n}+\hat{k}-\hat{m} \in B Z F_{N} \\ 0, & \text { if } \hat{n}-\hat{m} \notin B Z F \text { or } \hat{n}+\hat{k}-\hat{m} \notin B Z F_{N}\end{cases}
$$

hold, and that moreover

$$
\left\langle\hat{n}^{\prime}\left|H_{N}^{-}\right| \hat{n}\right\rangle=\sum_{\hat{k}} \sum_{\hat{m}} C(\hat{n}, \hat{k}, \hat{m}) E(\hat{k}, \hat{m}) \delta\left(\hat{n}^{\prime}, \hat{n}+\hat{k}-\hat{m}\right),
$$

where $\delta$ is the Kronecker symbol and where $E(\hat{k}, \hat{m})$ is defined by (3.6) (cf. also Remark 3.3).

From Lemma 3.5 one can draw the following

Conclusion 3.6. If $\hat{n}$ and $\hat{n}^{\prime}$ are given, $\left\langle\hat{n}^{\prime}\left|H_{N}^{-}\right| \hat{n}\right\rangle$ can be unequal zero only if $\hat{n}-\hat{m} \in B Z F_{N-2}$ and $\hat{n}^{\prime}-\hat{k} \in B Z F_{N-2}$. These relations can be satisfied only for $\left(\begin{array}{c}N \\ 2\end{array}\right)$ bzf $\hat{m}$ and $\hat{k}$. Hence the sums in (3.12) have finitely many summands.

3.2.2: Though the lemma is used in this paper solely in the above version, for later purposes a generalization of it will be proved in the next sections. (The expenditure is the same in both cases.) In order to do so, some notation is introduced.

Let $2 \leq M<N$ and let $A_{M}$ be a bounded operator defined on $\mathcal{H}_{M}^{-}$such that

$$
A_{N}=\Omega_{N}^{-}\left(A_{M}\right)
$$


is defined on $\mathcal{H}_{-}^{N}$. The matrix elements of $A_{M}$ with respect of $\mathcal{B}_{M}^{-}$are again denoted $E(\hat{k}, \hat{m})$ so that here

$$
\sum_{\alpha} k_{\alpha}=\sum_{\alpha} m_{\alpha}=M
$$

Moreover let

$$
\left\langle\hat{n}^{\prime}\left|A_{N}\right| \hat{n}\right\rangle:=\left\langle\Psi_{N}^{-}\left(\hat{n}^{\prime}\right), A_{N} \Psi_{N}^{-}(\hat{n})\right\rangle
$$

Finally, the function $C$ is defined as in Lemma 3.2 but with condition (3.14).

Proposition 3.7. The relation

$$
\left\langle\hat{n}^{\prime}\left|A_{N}\right| \hat{n}\right\rangle=\sum_{\hat{k}} \sum_{\hat{m}} C(\hat{n}, \hat{k}, \hat{m}) E(\hat{k}, \hat{m}) \delta\left(\hat{n}^{\prime}, \hat{n}+\hat{k}-\hat{m}\right)
$$

holds. (Cf. also Remark 3.3 and Conclusion 3.6.)

\subsection{Proof of Formula (3.16)}

3.3.1: The starting point is Formula (A.1.28) and the analogue to Formula (3.9). Thus

$$
\left\langle\hat{n}^{\prime}\left|A_{N}\right| \hat{n}\right\rangle=\left(\begin{array}{l}
N \\
M
\end{array}\right) \sum_{\hat{k}} \sum_{\hat{m}} E(\hat{k}, \hat{m}) Z\left(\hat{n}^{\prime}, \hat{n}+\hat{k}-\hat{m}\right)
$$

where

$$
Z\left(\hat{n}^{\prime}, \hat{n}, \hat{k}, \hat{m}\right)=\left\langle\Psi_{N}^{-}\left(\hat{n}^{\prime}\right),(T(\hat{k}, \hat{m}) \otimes 1 \otimes \cdots \otimes 1) \Psi_{N}^{-}(\hat{n})\right\rangle
$$

Here the operator $T(\hat{k}, \hat{m})$ is defined by strict analogy with (3.8). Thus

$$
\begin{aligned}
T(\hat{k}, \hat{m}) & =\Psi_{M}^{-}(\hat{k})\left\langle\Psi_{M}^{-}(\hat{m}), \cdot\right\rangle_{M} \\
& =(M !)^{-\frac{1}{2}} \sum_{Q \in \mathcal{S}_{M}} \sigma^{-}(Q) \Psi_{M}^{-}(\hat{k})\left\langle\phi_{\mu_{Q^{-1}(1)} \cdots \mu_{Q^{-1}(M)}}, \cdot\right\rangle_{M}
\end{aligned}
$$

where $\mu_{1}, \ldots, \mu_{M} \leftrightarrow \hat{m}$ with $\mu_{1}<\cdots<\mu_{M}$ is the correspondence defined by (A.1.13). Now using the correspondence $\nu_{1}, \ldots, \nu_{N} \leftrightarrow \hat{n}$ with $\nu_{1}<\cdots<\nu_{N}$ 
one finds that

$$
(T(\hat{k}, \hat{m}) \otimes 1 \otimes \cdots \otimes 1) \Psi_{N}^{-}(\hat{n})=\Psi_{M}^{-}(\hat{k}) \otimes \chi(\hat{n}, \hat{m})
$$

where

$$
\begin{aligned}
\chi(\hat{n}, \hat{m})= & (N ! M !)^{-\frac{1}{2}} \sum_{\substack{P \in \mathcal{S}_{N} \\
Q \in \mathcal{S}_{M}}} \sigma^{-}(P) \sigma^{-}(Q) \\
& \cdot\left(\prod_{j=1}^{M}\left\langle\phi_{\mu_{Q^{-1}(j)}}, \phi_{\nu_{P^{-1}(j)}}\right\rangle_{1}\right) \phi_{\nu_{P^{-1}(M+1)} \cdots \nu_{P^{-1}(N)}}
\end{aligned}
$$

Thus finally we obtain the relation

$$
Z\left(\hat{n}^{\prime}, \hat{n}, \hat{k}, \hat{m}\right)=\left\langle\Psi_{N}^{-}\left(\hat{n}^{\prime}\right), \Psi_{M}^{-}(\hat{k}) \otimes \chi(\hat{n}, \hat{m})\right\rangle .
$$

3.3.2: In this subsection the following proposition is proved:

$$
\chi(\hat{n}, \hat{m}) \neq 0
$$

if and only if $\hat{n}-\hat{m} \in B Z F$.

Firstly it is assumed that $\hat{n}-\hat{m} \notin B Z F$. Then there is a number $\alpha$ such that $m_{\alpha}=1$ and $n_{\alpha}=0$. Consequently, for each $Q \in \mathcal{S}_{M}$ there is an $r$ for which $Q^{-1}(r)=\alpha$ holds. But for each $P \in \mathcal{S}_{N}$ the relation $P^{-1}(r) \neq \alpha$ is true. Thus for each pair $P, Q$

$$
\prod_{j=1}^{M}\left\langle\phi_{\mu_{Q^{-1}(j)}}, \phi_{\nu_{P^{-1}(j)}}\right\rangle_{1}=0
$$

so that also $\chi(\hat{n}, \hat{m})=0$.

Secondly let us assume that $\hat{n}-\hat{m} \in B Z F$. Then for each $\alpha$ with $m_{\alpha}=1$ also $n_{\alpha}=1$ holds. Consequently one has to look for all pairs $Q, P$ such that

$$
\prod_{j=1}^{M}\left\langle\phi_{\mu_{Q^{-1}(j)}}, \phi_{\nu_{P^{-1}(j)}}\right\rangle_{1}=1 .
$$

For all other pairs $Q, P$ the product in (3.25) is zero because $\left\langle\phi_{\mu}, \phi_{\nu}\right\rangle_{1}=\delta_{\mu \nu}$. Thus (3.25) is equivalent to

$$
\mu_{Q^{-1}(j)}=\nu_{P^{-1}(j)}, \quad j=1, \ldots, M
$$


In order to satisfy (3.26), for a given $Q \in \mathcal{S}_{M}$ the permutation $P \in \mathcal{S}_{N}$ must be such that the $\mu_{1}, \ldots, \mu_{M}$, which by presumption occur in $\nu_{1}, \ldots, \nu_{M}$, occupy the places $1, \ldots, M$ being ordered by $Q$. All these pairs $Q, P$ can be explicitly indicated by the following procedure.

Let $S \in \mathcal{S}_{N}$ be that permutation for which

$$
\left(\nu_{S^{-1}(1)}, \ldots, \nu_{S^{-1}(N)}\right)=\left(\mu_{1}, \ldots, \mu_{M}, \varrho_{1}, \ldots, \varrho_{N-M}\right)
$$

where $\varrho_{1}, \ldots, \varrho_{N-M}$ are all those $\nu_{1}, \ldots, \nu_{N}$ which are unequal $\mu_{1}, \ldots, \mu_{M}$, and in addition let $\varrho_{1}<\cdots<\varrho_{N-M}$. Then with the help of (A.1.7) one finds

$$
\begin{aligned}
\Psi_{N}^{-}(\hat{n}) & =\sigma^{-}(S) \sqrt{N !} S_{N}^{-} U(S) \phi_{\nu_{1} \cdots \nu_{N}} \\
& =\sigma^{-}(S) \sqrt{N !} S_{N}^{-} \phi_{\mu_{1} \cdots \mu_{M} \varrho_{1} \cdots \varrho_{N-M}}
\end{aligned}
$$

so that $(3.21)$ now reads

$$
\begin{aligned}
\chi(\hat{n}, \hat{m})= & (N ! M !)^{-\frac{1}{2}} \sigma^{-}(S) \sum_{P, Q} \sigma^{-}(P) \sigma^{-}(Q) \\
& \cdot\left(\prod_{j=1}^{M}\left\langle\phi_{\mu_{Q^{-1}(j)}}, \phi_{\mu_{P^{-1}(j)}}\right\rangle\right) \phi_{\varrho_{P^{-1}(M+1)-M} \cdots \varrho_{P^{-1}(N-M)}} .
\end{aligned}
$$

It follows from (3.29) that only those pairs $Q, P$ give nonzero summands for which $P$ has the form:

$$
P=\left(Q, \begin{array}{c}
M+1, \ldots, N \\
M+1, \ldots, N
\end{array}\right)\left(\begin{array}{c}
1, \ldots, M \\
1, \ldots, M
\end{array}, R\right)
$$

where $R \in \mathcal{S}_{N-M}$ is an arbitrary permutation. Hence, for a given $Q$ there are $(N-M)$ ! permutations $P$ of the form (3.30) such that (3.26) is satisfied.

Since $R$ acts on $\left(\nu_{S^{-1}(M+1)}, \ldots, \nu_{S^{-1}(N)}\right)=\left(\varrho_{1}, \ldots, \varrho_{N-M}\right)$ one finally obtains

$$
\begin{aligned}
\chi(\hat{n}, \hat{m}) & =(N ! M !)^{-\frac{1}{2}} \sigma^{-}(S) \sum_{R Q} \sigma^{-}(R) \sigma^{-}(Q)^{2} \phi_{\varrho_{R^{-1}(1)} \cdots \varrho_{R^{-1}(N-M)}} \\
& =\left(\begin{array}{c}
N \\
M
\end{array}\right)^{-\frac{1}{2}} \sigma^{-}(S) \Psi_{N-M}^{-}(\hat{n}-\hat{m})
\end{aligned}
$$

for all pairs $\hat{n}, \hat{m}$ with $\hat{n}-\hat{m} \in B Z F$. Hence the proof of relation (3.23) is complete, and in addition the explicit form of $\chi(\hat{n}, \hat{m})$ is obtained. 
3.3.3: Inserting (3.31) into (3.20) and (3.18) yields

$$
Z\left(\hat{n}^{\prime}, \hat{n}, \hat{k}, \hat{m}\right)=\left(\begin{array}{c}
N \\
M
\end{array}\right)^{-\frac{1}{2}} \sigma^{-}(S)\left\langle\Psi_{N}^{-}\left(\hat{n}^{\prime}\right), S_{N}^{-}\left(\Psi_{M}^{-}(\hat{k}) \otimes \Psi_{N-M}(\hat{n}-\hat{m})\right)\right\rangle .
$$

With the help of (A.1.7) and by the correspondence $\hat{k} \leftrightarrow\left(\kappa_{1}, \ldots, \kappa_{M}\right)$ it follows that

$$
\begin{aligned}
& S_{N}^{-}\left(\Psi_{M}^{-}(\hat{k}) \otimes \Psi_{M-N}^{-}(\hat{n}-\hat{m})\right) \\
= & (M !(N-M) !)^{-\frac{1}{2}} S_{N}^{-}\left(\phi_{\kappa_{1} \cdots, \kappa_{M}} \otimes \phi_{\varrho_{1} \cdots \varrho_{N-M}}\right) \\
= & \left(\begin{array}{c}
N \\
M
\end{array}\right)^{-\frac{1}{2}} \sigma^{-}(T) \Psi_{N}^{-}(\hat{n}+\hat{k}-\hat{m}),
\end{aligned}
$$

where $T \in \mathcal{S}_{N}$ is the permutation which lines up the sequence $\left(\kappa_{1}, \ldots, \kappa_{M}\right.$, $\left.\varrho_{1}, \ldots, \varrho_{N-M}\right)$ in its natural order. Thus one obtains

$$
\begin{aligned}
& Z\left(\hat{n}^{\prime}, \hat{n}, \hat{k}, \hat{m}\right) \\
= & \left(\begin{array}{c}
N \\
M
\end{array}\right)^{-1} \sigma^{-}(S \cdot T)\left\langle\Psi_{N}^{-}\left(\hat{n}^{\prime}\right), \Psi_{N}^{-}(\hat{n}+\hat{k}-\hat{m})\right\rangle \\
= & \left(\begin{array}{c}
N \\
M
\end{array}\right)^{-1} \sigma^{-}(S \cdot T) \delta\left(\hat{n}^{\prime}, \hat{n}+\hat{k}-\hat{m}\right) .
\end{aligned}
$$

It follows that $Z\left(\hat{n}^{\prime}, \hat{n}, \hat{k}, \hat{m}\right) \neq 0$ exactly if $\hat{n}-\hat{m} \in B Z F, \hat{n}+\hat{k}-\hat{m} \in$ $B Z F_{N}$ and $\hat{n}^{\prime}=\hat{n}+\hat{k}-\hat{m}$.

3.3.4: Since the permutations $S$ and $T$ are uniquely defined by the sequences of indices $\left(\nu_{1}, \ldots, \nu_{N}\right),\left(\mu_{1}, \ldots, \mu_{M}\right)$ and $\left(\kappa_{1}, \ldots, \kappa_{M}\right)$ or equivalently by $\hat{n}, \hat{m}$ and $\hat{k}$ it is obvious to define the function $C$ by

$$
C(\hat{n}, \hat{k}, \hat{m})= \begin{cases}\sigma^{-}(T \cdot S)= \pm 1, & \text { if } \hat{n}-\hat{m} \in B Z F \text { and } \\ & \hat{n}+\hat{k}-\hat{m} \in B Z F_{N} \\ 0, & \text { otherwise. }\end{cases}
$$

Now inserting (3.34) together with (3.35) into (3.17) Formula (3.16) is seen to hold, thus also Lemma (3.5). With respect to the sums $\sum_{\hat{k}}$ and $\sum_{\hat{m}} \mathrm{I}$ refer to Remark 3.3 and Conclusion 3.6. 


\subsection{An algorithm for $C(\hat{n}, \hat{k}, \hat{m})$}

3.4.1: The question to be answered in this section reads: is there a finite procedure for calculating $C(\hat{n}, \hat{k}, \hat{m})$ if $\hat{n}, \hat{k}, \hat{m}$ are given $b z f$. As in Section 3.3 the more general case $2 \leq M<N$ is considered.

Since by definition $C(\hat{n}, \hat{k}, \hat{m})=0$ if the condition

$$
\hat{n}-\hat{m} \in B Z F \quad \text { and } \quad \hat{n}+\hat{k}-\hat{m} \in B Z F_{N}
$$

does not hold, only the case needs to be considered that (3.36) is true. Then

$$
C(\hat{n}, \hat{k}, \hat{m}):=\sigma^{-}(T \cdot S)=(-1)^{J(T)}(-1)^{J(S)},
$$

where $S$ is defined by (3.27) and $T$ by (3.33). Moreover, $J(P)$ here means the number of inversions of a permutation $P$ (cf. e.g. (A.1.6)).

3.4.2: To begin with, $J(S)$ is to be calculated. Let $\hat{n}$ be given. Then exactly $N$ numbers $\nu_{i}, i=1, \ldots, N$ exist such that $n_{\nu_{i}}=1$ and $\nu_{1}<\cdots<\nu_{N}$. Hence $\hat{n} \leftrightarrow\left(\nu_{1}, \ldots, \nu_{N}\right)$. Likewise, if $\hat{m}$ is given, exactly $M$ numbers $\mu_{j}, j=1, \ldots, M$ exist such that $m_{\mu_{j}}=1$ and $\mu_{1}<\cdots<\mu_{M}$.

Because of $\hat{n}-\hat{m} \in B Z F$ for each $j \in\{1, \ldots, M\}$ there is an $r_{j}$ such that

$$
\mu_{j}=\nu_{r_{j}} \quad \text { and } \quad j \leq r_{j}
$$

The permutation $S$ is defined by (3.27), i.e.

$$
\left.\left(\nu_{S^{-1}(1)}\right), \ldots, \nu_{S^{-1}(N)}\right)=\left(\mu_{1}, \ldots, \mu_{M}, \varrho_{1}, \ldots, \varrho_{N-M}\right)
$$

and $\varrho_{1}<\cdots<\varrho_{N-M}$. Then the right-hand side of (3.39) can be generated from $\left(\nu_{1}, \ldots, \nu_{N}\right)$ by the following procedure.

First, $\mu_{1}=\nu_{r_{1}}$ is positioned at the $r_{1}-t h$ place in $\left(\nu_{1}, \ldots, \nu_{N}\right)$. Therefore one needs $r_{1}-1$ inversions to bring $\mu_{1}$ at the first place. Thereby the positions of $\mu_{2}, \ldots, \mu_{M}$ in $\left(\nu_{1}, \ldots, \nu_{N}\right)$ are not changed.

Second, $\mu_{2}=\nu_{r_{2}}$ is positioned at the $r_{2}{ }^{t h}$ place in $\left(\nu_{1}, \ldots, \nu_{N}\right)$ so that one needs $r_{2}-2$ inversions to bring $\mu_{2}$ at the second place. Again the positions of $\mu_{3}, \ldots, \mu_{M}$ are not changed.

Thus, in order to bring $\mu_{j}=\nu_{r_{j}}$ to position $j$ one needs $r_{j}-j$ inversions. Therefore the total number of inversions, which realize the permutation $S$ 
in (3.39), is given by

$$
J(S)=\sum_{j=1}^{M} r_{j}-\frac{1}{2} M(M+1) .
$$

3.4.3: Now, $J(T)$ is to be determined. This task is the following. Let $\hat{n}-\hat{m}$ and $\hat{k}$ be given. Then $\hat{n}-\hat{m} \in B Z F$ corresponds to the sequence of indices $\left(\varrho_{1}, \ldots, \varrho_{N-M}\right)$ and $\hat{k}$ to the sequence $\left(\kappa_{1}, \ldots, \kappa_{M},\right)$. Thus the sequence of indices $\left(\kappa_{1}, \ldots, \kappa_{M}, \varrho_{1}, \ldots, \varrho_{N-M}\right)$, brought to its natural order by the permutation $T$ and denoted $\left(\nu_{1}^{\prime}, \ldots, \nu_{N}^{\prime}\right)$, corresponds to $\hat{n}+\hat{k}-\hat{m} \in B Z F$. Hence

$$
\left.\left(\nu_{T(1)}^{\prime}\right), \ldots, \nu_{T(N)}^{\prime}\right)=\left(\kappa_{1}, \ldots, \kappa_{M}, \varrho_{1}, \ldots, \varrho_{N-M}\right) .
$$

Therefore, because $\hat{n}+\hat{k}-\hat{m}$ is given, also $\left(\nu_{1}^{\prime}, \ldots, \nu_{N}^{\prime}\right)$ is determined so that for each $j \in\{1, \ldots, M\}$ the position $s_{j}$ of $\kappa_{j}$ in $\left(\nu_{1}^{\prime}, \ldots, \nu_{N}^{\prime}\right)$ can be read off with the help of the relations

$$
\kappa_{j}=\nu_{s_{j}}^{\prime} \text { and } j \leq s_{j} .
$$

Using the same arguments as in Section 3.4.2 one obtains for $T^{-1}$ the result

$$
J(T)=J\left(T^{-1}\right)=\sum_{j=1}^{M} s_{j}-\frac{1}{2} M(M+1) .
$$

3.4.4: Finally, the algorithm for $C(\hat{n}, \hat{k}, \hat{m})$ can be formulated thus:

$\mathbf{1}^{\text {st }}$ step: Take $b z f \hat{n}, \hat{k}$ and $\hat{m}$ which fulfil the equations $\sum n_{\alpha}=N$, $\sum k_{\alpha}=\sum_{\alpha} m_{\alpha}=M$, and test Condition (3.36). If it is satisfied go to the next step. If it is not, define $C(\hat{n}, \hat{k}, \hat{m})=0$, so that the task has been done.

$\mathbf{2}^{\text {nd }}$ step: Take $\hat{n}, \hat{m}$ and determine the corresponding sequences of indices $\left(\nu_{1}, \ldots, \nu_{N}\right)$ and $\left(\mu_{1}, \ldots, \mu_{M}\right)$. Then from (3.38) read off the numbers $r_{j}, j=1, \ldots, M$, and calculate $J(S)$ with the help of (3.40).

$\mathbf{3}^{\text {rd }}$ step: Take $\hat{n}+\hat{k}-\hat{m}$ and $\hat{k}$, and determine the corresponding sequences $\left(\nu_{1}^{\prime}, \ldots, \nu_{N}^{\prime}\right)$ and $\left(\kappa_{1}, \ldots, \kappa_{M}\right)$. Then from (3.42) read off the numbers $s_{j}, j=1, \ldots, M$ and calculate $J(T)$ with the help of (3.43).

$4^{\text {th }}$ step: Calculate $C(\hat{n}, \hat{k}, \hat{m})$ using (3.37).

The coefficients $C(\hat{n}, \hat{k}, \hat{m})$ do not depend on the specific physical system, for which they are used, rather they are completely combinatorial. In other words, they result solely from the algebraic structure imposed on the set 
$B Z F$. Therefore they can be computationally calculated once for all. A trivial special result is the following.

If $\hat{k}=\hat{m}$, then $T^{-1}=S$ so that

$$
C(\hat{n}, \hat{m}, \hat{m})=1 .
$$

\subsection{The final form of $\left\langle\hat{n}^{\prime}\left|H_{N}^{-}\right| \hat{n}\right\rangle$}

3.5.1: For the sake of simplicity in this section only the special case $M=2$ is considered. This does not entail any loss, because the Hamiltonians we are interested in this paper are supposed to have two-particle interactions. The starting point for this section therefore is (3.12). Moreover it is assumed that the matrix elements $E(\hat{k}, \hat{m})$ of the dummy Hamiltonian $H_{20}^{-}$and the coefficients $C(\hat{n}, \hat{k}, \hat{m})$ are given.

Now the problem to be solved reads as follows. Let the pair $\hat{n}^{\prime}, \hat{n}$ be given. Then determine those pairs $\hat{k}, \hat{m}$ for which the summands in $\sum_{\hat{k}}$ and $\sum_{\hat{m}}$ do not vanish on general grounds.

It is known from the previous considerations that for given $\hat{n}^{\prime}, \hat{n}$ only those $\hat{k}$ and $\hat{m}$ in (3.12) are relevant which satisfy the condition

$$
\hat{n}-\hat{m} \in B Z F, \quad \hat{n}+\hat{k}-\hat{m} \in B Z F_{N}, \quad \hat{n}^{\prime}=\hat{n}+\hat{k}-\hat{m},
$$

hence also

$$
\hat{n}^{\prime}-\hat{k} \in B Z F, \quad \hat{n}^{\prime}-\hat{n}=\hat{k}-\hat{m} .
$$

Consequently, the sums in (3.12) have only finitely many summands as already remarked in Conclusion 3.3.

3.5.2: In this section a disjoint dissection of all possible pairs $\hat{k}, \hat{m}$ for given $\hat{n}^{\prime}, \hat{n}$ will be defined. For this purpose it is useful to introduce some new

Notation 3.8. Let $\hat{k}, \hat{m} \in B Z F_{2}$ so that $\sum k_{\alpha}=\sum m_{\beta}=2$ is satisfied. Then the sequence $\hat{d}:=\hat{k}-\hat{m}$ is called differences sequence. The set of all difference sequences is denoted $\mathcal{D}$.

Consequently there are only three types $\mathcal{D}_{\varrho}, \varrho=0,1,2$ of possible $\hat{d}$ generated by $\hat{d}=\hat{k}-\hat{m}$ :

$\mathcal{D}_{0}$ contains only one element $\hat{o}:=(0, \ldots, 0, \ldots)$, and $\hat{o}$ is generated by all $\hat{k}, \hat{m}$ with $\hat{k}=\hat{m}$. 
$\mathcal{D}_{1}$ contains $\hat{d}=(1,-1,0, \ldots)$ and all permutations of it. They are generated by all $\hat{k}, \hat{m}$ such that for exactly one $\alpha$ the relation $k_{\alpha}=m_{\alpha}=1$ holds.

$\mathcal{D}_{2}$ contains $\hat{d}=(1,1,-1,-1,0, \ldots)$ and all permutations of it. They are generated by all $\hat{k}, \hat{m}$ for which no $\alpha$ exists such that $k_{\alpha}=m_{\alpha}=1$.

It follows immediately that the sets $\mathcal{D}_{0}, \mathcal{D}_{1}, \mathcal{D}_{2}$, are disjoint and that

$$
\mathcal{D}=\mathcal{D}_{0}, \cup \mathcal{D}_{1} \cup \mathcal{D}_{2}
$$

Many results of the next sections are based on the following

Proposition 3.9. For each pair $\hat{n}^{\prime}, \hat{n} \in B Z F_{N}$ there is a set

$$
\left\{\hat{d}_{1}, \ldots, \hat{d}_{L}\right\} \subset \mathcal{D}_{1}
$$

such that

$$
\hat{n}^{\prime}=\hat{n}+\hat{d}_{1}+\cdots+\hat{d}_{L}
$$

The number $L$ is uniquely determined by $\hat{n}^{\prime}$ and $\hat{n}$, but the difference sequences $\hat{d}_{j}, j=1, \ldots, L$ are not.

Proof. From the pair $\hat{n}^{\prime}, \hat{n}$ one forms the matrix

$$
X:=\left(\begin{array}{c}
\hat{n} \\
\hat{n}^{\prime}
\end{array}\right)=\left(\begin{array}{c}
n_{1}, n_{2}, \ldots \\
n_{1}^{\prime}, n_{2}^{\prime}, \ldots
\end{array}\right) .
$$

For each column of $X$ the following alternative holds:

$$
\left(\begin{array}{l}
n_{\varrho} \\
n_{\varrho}^{\prime}
\end{array}\right)=\left(\begin{array}{l}
0 \\
0
\end{array}\right) \text { or }\left(\begin{array}{l}
1 \\
1
\end{array}\right) \text { or }\left(\begin{array}{l}
0 \\
1
\end{array}\right) \text { or }\left(\begin{array}{l}
1 \\
0
\end{array}\right) \text {. }
$$

Because $\hat{n}$ and $\hat{n}^{\prime}$ both contain $N$ numbers 1 , there are equally many columns $\left(\begin{array}{l}0 \\ 1\end{array}\right)$ and $\left(\begin{array}{l}1 \\ 0\end{array}\right)$. Let $L$ by the number of each of the two kinds. Moreover let $\varrho_{j}$ and $\sigma_{j}, j=1, \ldots, L$ be numberings of the indices of these columns such that

$$
\left(\begin{array}{c}
n_{\varrho_{j}} \\
n_{\varrho_{j}}^{\prime}
\end{array}\right)=\left(\begin{array}{l}
0 \\
1
\end{array}\right) \quad \text { and } \quad\left(\begin{array}{l}
n_{\sigma_{j}} \\
n_{\sigma_{j}}^{\prime}
\end{array}\right)=\left(\begin{array}{l}
1 \\
0
\end{array}\right)
$$

Then for each pair of indices $\varrho_{j}, \sigma_{j}, j=1, \ldots, L$ define $\hat{d}_{j}=\left(d_{j 1}, d_{j 2}, \ldots\right)$ by $d_{j \varrho_{j}}=1, d_{j \sigma_{j}}=-1$ and $d_{j \alpha}=0, \alpha \neq \varrho_{j}, \sigma_{j}$. 
It follows from (3.52) that

$$
\hat{n}+\hat{d}_{j}=\left(\ldots, n_{\alpha}, \ldots, n_{\varrho_{j}}^{\prime}, \ldots, n_{\sigma_{j}}^{\prime}, \ldots, n_{\beta}, \ldots\right)
$$

if $\varrho_{j}<\sigma_{j}$, and analogously, if $\sigma_{j}<\varrho_{j}$.

Since $j \neq i$ implies $\varrho_{j} \neq \varrho_{i}, \sigma_{j} \neq \sigma_{i}$ the addition of $\hat{d}_{i}$ to $\hat{n}+\hat{d}_{j}$ can be carried through without altering $n_{\varrho_{j}}^{\prime}$ and $n_{\sigma_{j}}^{\prime}$ in (3.53). Thus, finally one ends up with (3.49) so that the proposition is proved.

Three immediate consequences are useful later on.

1.) For each pair $i, j$ with $i \neq j$ the relation $\hat{d}_{i}+\hat{d}_{j} \in \mathcal{D}_{2}$ holds.

2.) $\hat{n}^{\prime}-\hat{n} \in \mathcal{D}_{\varrho}$, exactly if $L=\varrho, \varrho=0,1,2$.

3.) $\hat{n}^{\prime}-\hat{n} \notin \mathcal{D}$ exactly if $L \geq 3$.

3.5.3: Using the results of the previous sections the problem formulated in 3.5.1 now can be solved by giving a disjoint classification of the matrix elements defined by (3.12). According to (3.47) four cases have to be taken into account.

$\mathbf{1}^{\text {st }}$ case: $\hat{n}^{\prime}-\hat{n} \notin \mathcal{D}$. It follows immediately from (3.12) that

$$
\left\langle\hat{n}^{\prime}\left|H_{N}^{-}\right| \hat{n}\right\rangle=0
$$

$\mathbf{2}^{n d}$ case: $\hat{n}^{\prime}-\hat{n} \in \mathcal{D}_{0}$, i.e. $\hat{n}^{\prime}=\hat{n}$. Then $\left\langle\hat{n}\left|H_{N}^{-}\right| \hat{n}\right\rangle$ is unequal zero only, if also $\hat{k}=\hat{m}$. Hence the double sum $\sum_{\hat{k}} \sum_{\hat{m}}$ reduces to a single sum $\sum_{\hat{m}}$. This sum runs over all $\hat{m}$ for which $\hat{n}-\hat{m} \in B Z F$ holds. Since $\hat{n}$ contains $N$ numbers 1 there are exactly $\left(\begin{array}{c}N \\ 2\end{array}\right)$ different sequences $\hat{m}$ such that this condition is satisfied. Because of $C(\hat{n}, \hat{m}, \hat{m})=1$, define

$$
\mathcal{E}(\hat{n}, \hat{o})=\sum_{\hat{m}} E(\hat{m}, \hat{m})
$$

for all $\hat{m}$ with $\hat{n}-\hat{m} \in B Z F$. Thus finally

$$
\left\langle\hat{n}\left|H_{N}^{-}\right| \hat{n}\right\rangle=\mathcal{E}(\hat{n}, \hat{o}) .
$$

$\mathbf{3}^{r d}$ case: $\hat{n}^{\prime}-\hat{n}=\hat{d}_{1} \in \mathcal{D}_{1}$. Hence $\hat{n}+\hat{d}_{1} \in B Z F$. Then $\left\langle\hat{n}^{\prime}\left|H_{N}^{-}\right| \hat{n}\right\rangle$ is unequal zero only, if $\hat{k}=\hat{m}+\hat{d}_{1}$. Therefore the double sum $\sum_{\hat{k}} \sum_{\hat{m}}$ again reduces to a single sum $\sum_{\hat{m}}$ which runs over all $\hat{m}$ so that $\hat{n}-\hat{m} \in B Z F$ and $\hat{m}+\hat{d}_{1} \in B Z F$. These $\hat{m}$ can be characterized as follows.

Let $\hat{d}_{1}$ be given by $d_{1 \kappa}=1, d_{1 \mu}=-1$ and $d_{1 \beta}=0, \beta \neq \kappa, \mu$. Hence $n_{\kappa}=0$ and $n_{\mu}=1$. Then $\hat{m}+\hat{d}_{1} \in B Z F$ if and only if $m_{\kappa}=0$ and $m_{\mu}=1$. In 
order to satisfy the condition $\hat{n}-\hat{m} \in B Z F$ it is necessary and sufficient that $n_{\mu}=1$ and that there is an $\alpha \neq \kappa, \mu$, for which $n_{\alpha}=1$ and $m_{\alpha}=1$ holds. Since $n_{\kappa}=0$ and $n_{\mu}=1$ there are $N-1$ numbers $\alpha \neq \kappa, \mu$ for which $n_{\alpha}=1$ so that $\sum_{\hat{m}}$ runs over all $\hat{m}$ for which $m_{\alpha}=m_{\mu}=1$. Now define

$$
\mathcal{E}\left(\hat{n}, \hat{d}_{1}\right)=\sum_{\hat{m}} C\left(\hat{n}, \hat{m}+\hat{d}_{1}, \hat{m}\right) E\left(\hat{m}+\hat{d}_{1}, \hat{m}\right) .
$$

Then

$$
\left\langle\hat{n}^{\prime}\left|H_{N}^{-}\right| \hat{n}\right\rangle=\mathcal{E}\left(\hat{n}, \hat{d}_{1}\right)
$$

if $\hat{n}^{\prime}=\hat{n}+\hat{d}_{1}, \hat{d}_{1} \in \mathcal{D}_{1}$

$4^{\text {th }}$ case: $\hat{n}^{\prime}-\hat{n}=\hat{d}_{2} \in \mathcal{D}_{2}$. Let $\hat{d}_{2}$ be defined by $d_{2 \kappa}=d_{2 \lambda}=1, d_{2 \mu}=$ $d_{2 \nu}=-1$. Then $\hat{k}$ and $\hat{m}$ with $\hat{k}-\hat{m}=\hat{d}_{2}$ are uniquely determined by $k_{\kappa}=$ $k_{\lambda}=1$ and $m_{\mu}=m_{\nu}=1$. Hence the $\sum_{\hat{h}} \sum_{\hat{m}}$ reduces to a single term. Now define

$$
\mathcal{E}\left(\hat{n}, \hat{d}_{2}\right)=C\left(\hat{n}, \hat{m}+\hat{d}_{2}, \hat{m}\right) E\left(\hat{m}+\hat{d}_{2}, \hat{m}\right)
$$

where $\hat{m}$ is determined by $m_{\mu}=m_{\nu}=1$. Then, if $\hat{n}^{\prime}-\hat{n}=d_{2} \in \mathcal{D}_{2}$

$$
\left\langle\hat{n}^{\prime}\left|H_{N}^{-}\right| \hat{n}\right\rangle=\mathcal{E}\left(\hat{n}, \hat{d}_{2}\right) .
$$

3.5.4: Summing up one obtains

Proposition 3.10. 1.) The matrix elements of the fermionic Hamiltonian $H_{N}^{-}$defined in Assumption 3.1 (Section 3.1.1.) are given by

$$
\left\langle\hat{n}^{\prime}\left|H_{N}^{-}\right| \hat{n}\right\rangle= \begin{cases}\mathcal{E}\left(\hat{n}, \hat{n}^{\prime}-\hat{n}\right), & \text { if } \quad \hat{n}^{\prime}-\hat{n} \in \mathcal{D} \\ 0, & \text { if } \quad \hat{n}^{\prime}-\hat{n} \notin \mathcal{D}\end{cases}
$$

and

$$
\mathcal{E}\left(\hat{n}^{\prime}, \hat{n}-\hat{n}^{\prime}\right)=\overline{\mathcal{E}}\left(\hat{n}, \hat{n}^{\prime}-\hat{n}\right)
$$

2.) Let be given $\hat{n} \in B Z F$ and $\hat{d} \in \mathcal{D}$ so that $\hat{n}+\hat{d} \in B Z F$. Then it follows from Formula (3.12) that

$$
\mathcal{E}(\hat{n}, \hat{d})=\sum_{\hat{m}} C(\hat{n}, \hat{m}+\hat{d}, \hat{m}) E(\hat{m}+\hat{d}, \hat{m}),
$$

where the sum runs over all $\hat{m}$ for which $\hat{n}-\hat{m} \in B Z F$ and $\hat{m}+\hat{d}=: \hat{k} \in$ $B Z F_{2}$. Hence, the matrix elements $\left\langle\hat{n}^{\prime}\left|H_{N}^{-}\right| \hat{n}\right\rangle$ are determined solely by the 
matrix elements $E(\hat{k}, \hat{m})$ of $H_{20}^{-}$, for which $\hat{k}-\hat{m}=\hat{d}=\hat{n}^{\prime}-\hat{n}$. The sum in (3.63) is finite.

For bosons a result holds, which is formally equal to (3.12), (3.61) and 3.63), but the terms $C, E, \mathcal{E}$ are defined differently.

\section{Heuristic Considerations}

4.1: The result of Section 3, summarized in Formula (3.61), is completely formal up to now. This is due to two unsolved questions connected with it. They read as follows.

First, can Assumption 3.1 be verified? More concretely, is it possible to find a one-particle Hilbert space $\mathcal{H}^{1}$ such that the dummy Hamiltonian $\mathrm{H}_{20}^{-}$ is defined on $\mathcal{H}_{-}^{2}$ and is bounded, and such that in addition the spectrum of $H_{20}^{-}$contains the discrete eigenvalues of the dummy Hamiltonian $\bar{H}_{20}^{-}$, which is primarily defined? (cf. e.g. (A.2.1), (A.2.4), (A.2.6).)

Second, is Formula (3.61) of any advantage for the spectral problem of $H_{N}^{-}$?

4.2: To begin with, let us look for an answer to the first question. As is already described in Subsection 2.1, any investigation of the Hamiltonian $\bar{H}_{N}$ of an $N$-particle system starts with a more or less informal specification of the external fields acting upon the particles and of their interactions. Customarily this is done using the position-spin representation. Then $\bar{H}_{N}$ is of the form (1.1) or, what is the same, (2.1). It is densely defined in a Hilbert space $\overline{\mathcal{H}}^{N}$. Likewise the corresponding dummy Hamiltonian $\bar{H}_{20}^{-}$can be immediately written down as is shown for two examples in Appendix A.2. It is defined in a dense linear submanifold of $\overline{\mathcal{H}}^{2}$.

In order to verify Assumption 3.1 the space $\overline{\mathcal{H}}^{1}$ has to be properly restricted to a subspace $\mathcal{H}^{1}$. Such a restriction in turn can be carried through by finding a proper orthonormal system $\mathcal{O}_{1}$ in $\overline{\mathcal{H}}^{1}$ so that $\mathcal{H}^{1}=\operatorname{span} \mathcal{O}_{1}$. Having in mind the physical meaning of $\mathcal{H}^{1}$ suggests taking the HartreeFock procedure for $\bar{H}_{20}^{-}$to determine $\mathcal{O}_{1}$. (For the details cf. Appendix A.3.) This is because this procedure is based on the Ritz variational principle which guarantees optimal approximation. Disregarding the fact that the Hartree-Fock procedure generally is an infinite task, let us assume that it is completely carried through for the dummy Hamiltonian $\bar{H}_{20}^{-}$in $\overline{\mathcal{H}}_{-}^{2}$. Thus one has obtained $\mathcal{O}_{1}$ and an orthonormal system $\mathcal{O}_{2} \subset \overline{\mathcal{H}}_{-}^{2}$ of vectors

$$
\Psi_{\kappa \lambda}^{-}=\frac{1}{\sqrt{2}}\left(\phi_{\kappa} \otimes \phi_{\lambda}-\phi_{\lambda} \otimes \phi_{\kappa}\right), \quad \kappa<\lambda .
$$


By definition, $\mathcal{O}_{1}$ is an ONB of $\mathcal{H}^{1}$, therefore $\mathcal{O}_{2}$ is an ONB of $\mathcal{H}_{2}^{-}$. The corresponding energy levels $E_{\kappa \lambda}=E(\hat{m}, \hat{m})$ for $\kappa, \lambda \leftrightarrow \hat{m}$ approximate the discrete eigenvalues of $\bar{H}_{20}^{-}$outside its continuous spectrum. Since the Hamiltonians considered in this paper are supposed to have a bounded discrete spectrum, the set of the energy levels $E_{\kappa \lambda}$ is also bounded.

Then the restriction $H_{20}^{-}$of $\bar{H}_{20}^{-}$to the space $\mathcal{H}_{-}^{2}$ is bounded because its spectrum is approximated by the set $\left\{E_{\kappa \lambda}: \kappa<\lambda\right\}$ and its eigenvectors by the set $\mathcal{O}_{2}$. As usual $H_{20}^{-}$can be defined on the whole space $\mathcal{H}_{-}^{2}$ using its matrix representation with respect to the ONB $\mathcal{O}_{2}$.

In most cases of practical application the complete Hartree-Fock procedure cannot be achieved, because it is infinite. Therefore one has to content oneself with a finite section of this procedure. But also such a finite procedure can be complicated.

Thus other methods were invented which are equivalent to the HartreeFock procedure or approximate it.

4.3: Let us now come to the second question. From (3.61) one draws immediately a simple consequence.

Proposition 4.1. The matrix of the Hamiltonian $H_{N}^{-}=\Omega_{N}^{-}\left(H_{20}^{-}\right)$is diagonal if the matrix of the dummy Hamiltonian $H_{20}^{-}$is diagonal.

Unfortunately this result cannot be used to obtain the exact discrete eigenvalues of a realistic $N$-particle system, because the exact eigenvectors of a dummy Hamiltonian $H_{20}^{-}$containing interaction are not elements of any ONB $\mathcal{B}_{2}^{-}$. But, if one contents with a Hartree-Fock approximation of the eigenvalues of $H_{20}^{-}$, Proposition 4.1 delivers a Hartree-Fock-like approximation for the eigenvalues of $H_{N}^{-}$.

Thus, if one wants to obtain better approximations, one has to solve the following problem. Since the results of Section 3 are valid for arbitrary orthonormal bases $\mathcal{B}_{1} \subset \mathcal{H}^{1}$, one firstly has to choose such an ONB and one has to calculate the matrix elements $E(\hat{k}, \hat{m})$ of $\mathcal{H}_{20}^{-}$for the ONB $\mathcal{B}_{2}^{-}$. The second part of the problem then is the question, wether the choise of $\mathcal{B}_{1}$ is helpful for a reasonable approximation of $\mathcal{H}_{20}^{-}$and also of $\mathcal{H}_{N}^{-}$.

A heuristic idea to cope with this problem is the following. Choose the ONBs $\mathcal{B}_{1}$, resp. $\mathcal{B}_{2}^{-}$such that the matrix $E$ of $H_{20}^{-}$with respect to $\mathcal{B}_{2}^{-}$, i.e. the matrix defined by the elements $E(\hat{k}, \hat{m})$ is "as diagonal as possible". This means the elements of $\mathcal{B}_{2}^{-}$should approximate the eigenvectors of $H_{20}^{-}$ optimally. Hence we end up again with the Hartree-Fock method, an equivalent of it or an approximation. Therefore $\mathcal{B}_{1}=\mathcal{O}_{1}$ and $\mathcal{B}_{2}^{-}=\mathcal{O}_{2}$ with $\mathcal{O}_{1}$ and $\mathcal{O}_{2}$ being defined in Section 4.2 and in Appendix 3. 
In order to get further insight into the general structure of the matrix $E$ I will give some purely heuristic arguments, which are based on physical intuition. For this purpose let the Hartree-Fock energy levels $E_{\mu \lambda}$ be numbered such that $E_{\mu \nu} \leq E_{\mu \lambda}$ if $\mu<\nu<\lambda$. Then, if $\lambda$ is large enough, i.e. if it exceeds a certain value $\bar{\alpha}$ one expects that the particle having state $\lambda$ is "almost" free, so that the interaction between the two particles having the states $\mu$ and $\lambda$ is "almost" zero. Thus the two particles with states $\mu$ and $\lambda$ are "almost" free, if one particle of this pair is "almost" free. This implies, that the vector $\Psi_{\mu \lambda}^{-} \in \mathcal{H}_{-}^{2}$ is "almost" an eigenvector of $H_{20}^{-}$. Now let $\mu, \lambda$ correspond to a sequence of occupation numbers $\hat{m}$. Then $E(\hat{m}, \hat{m})$ is "almost" an eigenvalue of $H_{20}^{-}$for the eigenvector $\Psi_{\kappa \lambda}^{-}=\Psi_{2}^{-}(\hat{m})$, so that $E(\hat{k}, \hat{m})$ is "almost" equal to $E(\hat{m}, \hat{m}) \delta(\hat{k}, \hat{m})$. Consequently $E(\hat{k}, \hat{m})$ is "small", i.e. it is "almost" zero, if $\hat{k} \neq \hat{m}$.

Now let us suppose that the term "small" has been concretized. Then the above considerations can be summarized in the following assumption.

There is a natural number $\bar{\alpha}$ such that $E(\hat{k}, \hat{m})$ is small for each pair $\hat{k}, \hat{m}$ with $\hat{k} \neq \hat{m}$, for which a $k_{\sigma}=1, \sigma>\bar{\alpha}$ exists or an $m_{\varrho}=1, \varrho>\bar{\alpha}$.

This suggests truncating the matrix $E$ by substituting zeros for its small elements. Then intuitively one conjectures that the truncated matrix leads to an approximate solution of the spectral problem of $H_{N}^{-}$which is the goal of this paper. The precise meaning of the conjecture will be given in Section 5.1.

4.4: The first step is defining the truncated dummy Hamiltonian $\hat{H}_{20}^{-}$and drawing some consequences. The operator $\hat{H}_{20}^{-}$is determined by its matrix $\hat{E}$, which is given by the elements:

$$
\hat{E}(\hat{k}, \hat{m})= \begin{cases}0, & \text { if } \hat{k} \neq \hat{m} \text { and if } \sigma, \varrho \text { exist such that } \\ & k_{\sigma}=1, \sigma>\bar{\alpha} \text { or } m_{\varrho}=1, \varrho>\bar{\alpha} \\ E(\hat{k}, \hat{m}), & \text { otherwise. }\end{cases}
$$

According to this definition $\hat{E}$ is a finite nondiagonal matrix of order $\left(\begin{array}{c}\bar{\alpha} \\ 2\end{array}\right)$ with an infinite diagonal tail of elements $E(\hat{m}, \hat{m})$ where $\hat{m}$ contains at least one $m_{\varrho}=1, \varrho>\hat{\alpha}$.

Consequently, $\hat{H}_{20}^{-}$is bounded so that $\hat{H}_{N}^{-}=\Omega_{N}^{-}\left(\hat{H}_{20}^{-}\right)$is also bounded and has the form $(3.61)$, but with $\hat{\mathcal{E}}(\hat{n}, \hat{d})$ defined by $\hat{E}(\hat{k}, \hat{m})$ the same way as $\mathcal{E}(\hat{n}, \hat{d})$ is determined by $E(\hat{k}, \hat{m})$. Then we obtain the following

Proposition 4.2. Let us consider $\left\langle\hat{n}^{\prime}\left|\hat{H}_{N}^{-}\right| \hat{n}\right\rangle$. 
1.) If $\hat{n}^{\prime}-\hat{n} \notin \mathcal{D}$, one concludes from (3.12) that

$$
\left\langle\hat{n}^{\prime}\left|\hat{H}_{N}^{-}\right| \hat{n}\right\rangle=\left\langle\hat{n}^{\prime}\left|H_{N}^{-}\right| \hat{n}\right\rangle=0
$$

2.) If $\hat{n}^{\prime}-\hat{n}=\hat{d} \in \mathcal{D}$ and if there is $\lambda>\bar{\alpha}$ such that $d_{\lambda} \neq 0$, it follows from $\hat{d}=\hat{k}-\hat{m}$ that $k_{\lambda}=1$ or $m_{\lambda}=1$. Hence $\hat{E}(\hat{k}, \hat{m})=0$, and consequently

$$
\left\langle\hat{n}^{\prime}\left|\hat{H}_{N}^{-}\right| \hat{n}\right\rangle=0
$$

3.) Now let $\hat{n}^{\prime}-\hat{n}=\hat{d} \in \mathcal{D}$ and suppose that $d_{\lambda}=0$, if $\lambda>\bar{\alpha}$. Then if $\hat{d} \in \mathcal{D}_{2}$, the bzf $\hat{k}$ and $\hat{m}$ are uniquely determined and one obtains (cf. Formula (3.59))

$$
\left\langle\hat{n}^{\prime}\left|\hat{H}_{N}^{-}\right| \hat{n}\right\rangle=\hat{\mathcal{E}}(\hat{n}, \hat{d})=\mathcal{E}(\hat{n}, \hat{d})=\left\langle\hat{n}^{\prime}\left|H_{N}^{-}\right| \hat{n}\right\rangle
$$

If $\hat{d} \in \mathcal{D}_{1}$, things are more complicated. One has to apply the general method described in Section 3.5.3 for several special cases. The matrix element $\left\langle\hat{n}^{\prime}\left|\hat{H}_{N}^{-}\right| \hat{n}\right\rangle$ can be zero or unequal zero, and it need not be equal to $\left\langle\hat{n}^{\prime}\left|H_{N}^{-}\right| \hat{n}\right\rangle$.

4.5: By these considerations the problem of determining the eigenvalues of $H_{N}^{-}$is transformed into the following two ones.

First, do the eigenvalues of $\hat{H}_{N}^{-}$approximate those of $H_{N}^{-}$?

Second, can the eigenvalues of $\hat{H}_{N}^{-}$be calculated, at least partially?

The first problem is treated in Section 5, the second one in Section 6 .

\section{Spectral Approximation}

5.1: The dummy operators $H_{20}^{-}$and $\hat{H}_{20}^{-}$are defined on $\mathcal{H}_{-}^{2}$ and are bounded so that $D:=H_{20}^{-}-\hat{H}_{20}^{-}$is also bounded and defined on $\mathcal{H}_{-}^{2}$. Moreover $\hat{H}_{20}^{-}$ and $D$ depend on the fixed number $\bar{\alpha}$. In what follows these operators are understood to be functions of a parameter $\alpha \in \mathbb{N}$ and $\alpha \geq 2$ so that they are written $D(\alpha)$ and $\hat{H}_{20}^{-}(\alpha)$, and for the matrix elements of $\hat{E}$ we write $\hat{E}_{\alpha}(\hat{k}, \hat{m})$. Then by definition

$$
\langle\hat{k}|D(\alpha)| \hat{m}\rangle=E(\hat{k}, \hat{m})-\hat{E}_{\alpha}(\hat{k}, \hat{m}), \quad \alpha \geq 2
$$


Therefore

$$
\langle\hat{k}|D(\alpha)| \hat{m}\rangle= \begin{cases}0, & \text { if } \hat{k}=\hat{m} \text { or } \hat{k} \leftrightarrow \kappa_{1}, \kappa_{2} \leq \alpha \\ \langle\hat{k}|D(2)| \hat{m}\rangle, & \text { and } \hat{m} \leftrightarrow \mu_{1}, \mu_{2} \leq \alpha, \\ \kappa_{1}>\alpha \text { or } \kappa_{2}>\alpha \text { or } \mu_{1}>\alpha \text { or } \mu_{2}>\alpha .\end{cases}
$$

These properties have the following consequence.

Proposition 5.1. The sequence $(D(\alpha): \alpha \in \mathbb{N}, \alpha \geq 2)$ converges strongly to 0 .

Proof. Let the projections $F_{\alpha}$ and $F_{\alpha}^{\prime}$ be defined by

$$
F_{\alpha}=\sum_{\kappa_{1}, \kappa_{2}>\alpha}^{\infty} \Psi_{\kappa_{1} \kappa_{2}}^{-}\left\langle\Psi_{\kappa_{1}, \kappa_{2}}^{-}, \cdot\right\rangle
$$

and $F_{\alpha}^{\prime}=1-F_{\alpha}$. Then $F_{\alpha}$ converges strongly to 0 , if $\alpha \rightarrow \infty$, and $F_{\alpha}^{\prime}$ to 1 . By a simple calculation using Formula (5.2) one verifies that for each $f$ $\in \mathcal{H}_{-}^{2}$ :

$$
\begin{aligned}
& F_{\alpha} D(\alpha) f=F_{\alpha} D(2) f, \\
& F_{\alpha}^{\prime} D(\alpha) f=F_{\alpha}^{\prime} D(2) F_{\alpha} f .
\end{aligned}
$$

Therefore

$$
\|D(\alpha) f\| \leq\left\|F_{\alpha} D(2) f\right\|+\left\|F_{\alpha}^{\prime} D(2) F_{\alpha} f\right\|
$$

Because of

$$
\left\|F_{\alpha}^{\prime} D(2) F_{\alpha} f\right\| \leq\|D(2)\|\left\|F_{\alpha} f\right\|
$$

and because $F_{\alpha}$ converges to 0 , the proposition is seen to hold.

Now, in order to transfer the last result to $\hat{H}_{N}^{-}(\alpha):=\Omega_{N}^{-}\left(\hat{H}_{20}(\alpha)\right)$ one needs the following theorem.

Proposition 5.2. Let be given a sequence $\left(A_{M}(r): r \in \mathbb{N}\right)$ of bounded operators defined on $\mathcal{H}^{\mathcal{M}}$. If $A_{M}(r)$ converges strongly to $0_{M}$ for $r \rightarrow \infty$, then also

$$
s-\lim _{r-\infty} \Omega_{N}^{-}\left(A_{M}(r)\right)=0_{N}
$$

i.e. $\Omega_{N}^{-}\left(A_{M}(r)\right)$ converges strongly. 
Proof. 1.) Let $g \in \mathcal{H}^{N}$, then

$$
g=\sum_{\kappa_{1} \cdots \kappa_{N}} b_{\kappa_{1} \cdots \kappa_{N}} \phi_{\kappa_{1} \cdots \kappa_{N}}
$$

If

$$
\chi_{\kappa_{M+1} \cdots \kappa_{N}}:=\sum_{\kappa_{1} \cdots \kappa_{M}} b_{\kappa_{1} \cdots \kappa_{N}} \phi_{\kappa_{1} \cdots \kappa_{M}},
$$

it follows that

$$
g=\sum_{\kappa_{M+1} \cdots \kappa_{N}} \chi_{\kappa_{M+1} \cdots \kappa_{N}} \otimes \phi_{\kappa_{M+1} \cdots \kappa_{N}}
$$

and

$$
\|g\|^{2}=\sum_{\kappa_{M+1} \cdots \kappa_{N}}\left\|\chi_{\kappa_{M+1} \cdots \kappa_{N}}\right\|^{2} .
$$

If $B$ is a bounded operator on $\mathcal{H}^{M}$, then

$$
\|(B \otimes 1 \otimes \cdots \otimes 1) g\|^{2}=\sum_{\kappa_{M+1} \cdots \kappa_{N}}\left\|B \chi_{\kappa_{M+1} \cdots \kappa_{N}}\right\|^{2} .
$$

2.) Now, let us consider the operators $A_{M}(r)$. By supposition $A_{M}(r)$ converges strongly to $0_{M}$. Hence by the principle of uniform boundedness (cf. e.g. [13], p. 150) there is a number $K$ such that for all $r \in \mathbb{N}$ :

$$
\left\|A_{M}(r)\right\| \leq K
$$

Thus, one obtains for all $r \in \mathbb{N}$ :

$$
\sum_{\kappa_{M+1} \cdots \kappa_{N}}\left\|A_{M}(r) \chi_{\kappa_{M+1} \cdots \kappa_{N}}\right\|^{2} \leq K^{2} \sum_{\kappa_{M+1} \cdots \kappa_{N}}\left\|\chi_{\kappa_{M+1} \ldots \kappa_{N}}\right\|^{2} .
$$

Hence, by the criterion of Weierstraß the left series converges uniformly with respect to the variable $r$. Therefore the limit $r \rightarrow \infty$ can be interchanged with the sum so that

$$
\begin{aligned}
& \lim _{r \rightarrow \infty}\left\|\left(A_{M}(r) \otimes 1 \otimes \cdots \otimes 1\right) g\right\|^{2} \\
= & \sum_{\kappa_{M+1} \ldots \kappa_{N}} \lim _{r \rightarrow \infty}\left\|A_{M}(r) \chi_{\kappa_{M+1} \cdots \kappa_{N}}\right\|^{2}=0 .
\end{aligned}
$$

Thus, because for all $g \in \mathcal{H}_{-}^{N}$ the relation

$$
\left\|\Omega_{N}^{-}\left(A_{M}(r)\right) g\right\| \leq\left(\begin{array}{c}
N \\
M
\end{array}\right)\left\|\left(A_{M}(r) \otimes 1 \otimes \cdots\right) g\right\|
$$

holds, Formula (5.7) is proved. 
Then, with the help of the Propositions 5.1 and 5.2 one obtains the following consequences.

Proposition 5.3. 1.) The sequence $\left(\hat{H}_{N}^{-}(\alpha): \alpha \in \mathbb{N}, \alpha \geq 2\right)$ converges strongly to $H_{N}^{-}$. In other words, the operators $\hat{H}_{N}^{-}(\alpha)$ approximate $H_{N}^{-}$(cf. [12], p. 228, Formula 5.1). Hence all results concerning the approximation of one operator by a strongly converging sequence of operators are valid for $H_{N}^{-}$and $\hat{H}_{N}^{-}(\alpha)$. (cf. [12], Chapter 5.). Here only two of these properties are sketched.

2.) If $e$ is an isolated eigenvalue of $H_{N}^{-}$, then there is a sequence $\left(e_{\alpha}\right.$ : $\alpha \in \mathbb{N}$ ) such that $e_{\alpha}$ is an eigenvalue of $\hat{H}_{N}^{-}(\alpha)$ and such that $e_{\alpha} \rightarrow e$. (cf. [12], p. 239, Theorem 5.12).

3.) Let $P$ be the spectral projection belonging to an eigenvalue e of $\mathrm{H}_{N}^{-}$. Then an $\alpha_{0}$ exists such that for each $\alpha>\alpha_{0}$ there is a spectral projection $P_{\alpha}$ belonging to $\hat{H}_{N}^{-}(\alpha)$ and such that $P_{\alpha}$ converges strongly to $P$ for $\alpha \rightarrow \infty$. (cf. [12], p. 240, Theorem 5.13.)

5.2: Besides the approximation of $H_{N}^{-}$by $\hat{H}_{N}^{-}(\alpha)$ sketched above there are results, which are based on the norm of $D(\alpha)$, i.e. on $\delta(\alpha):=\|D(\alpha)\|$. Using the Formulae (A.1.29) and (A.1.30) one obtains the relation

$$
\left\|H_{N}^{-}-\hat{H}_{N}^{-}(\alpha)\right\|=\left\|\Omega_{N}^{-}(D(\alpha))\right\| \leq\left(\begin{array}{c}
N \\
2
\end{array}\right) \delta(\alpha) .
$$

Thus it follows that $\hat{H}_{N}^{-}(\alpha)$ converges in norm to $H_{N}^{-}$if

$$
\lim _{\alpha \rightarrow \infty} \delta(\alpha)=0
$$

Theorems concerning spectral approximation based on convergence in norm can be found in [12], p. 291, Theorem 4.10, p. 362, Theorem 5.10 and in [13], p. 249, Proposition 5.28.

\section{The operator $\hat{H}_{N}^{-}$and its matrix}

\subsection{Preliminary remarks}

6.1.1: The aim of this section is proving

Proposition 6.1. 1.) The operator $\hat{H}_{N}^{-}$defined on $\mathcal{H}_{-}^{N}$ is an orthogonal sum of operators defined on finite dimensional (orthogonal) subspaces of $\mathcal{H}_{-}^{N}$. 
2.) Moreover, using the matrix of $\hat{H}_{N}^{-}$the matrices of the suboperators can be determined explicitly.

If this proposition is verified, we have obtained a block-diagonalization of $\hat{H}_{N}^{-}$. Thus a way is opened, depending on the numbers $\bar{\alpha}$ and $N$, to calculate the eigenvalues of $\hat{H}_{N}^{-}$with the help of numerical methods. Purely analytical solutions of the eigenvalue problem of $\hat{H}_{N}^{-}$are also possible, if $\bar{\alpha}=2,3,4$. But, in these cases one cannot expect that $\hat{H}_{N}^{-}$is a good approximation of a realistic Hamiltonian $H_{N}^{-}$.

6.1.2: In order to verify Proposition 6.1 some further notation is used, which is provided by

Definition 6.2. 1.) Let be given an $\hat{n} \in B Z F_{N}$ and a natural number $\alpha$, which for the moment is completely arbitrary. Then

$$
(\hat{n}, \alpha):=\left(n_{1}, \ldots, n_{\alpha}\right) \quad \text { and } \quad(\alpha, \hat{n}):=\left(n_{\alpha+1}, n_{\alpha+2}, \ldots\right)
$$

For the infinite second part of $\hat{n}$ also the abbreviation $(\alpha, \hat{n})=: \hat{r}$ is used.

2.) Let $\hat{r}$ be given. Then $\mathcal{N}_{\beta}(\hat{r})$ denotes the set of all $\hat{n} \in B Z F_{N}$, for which $(\alpha, \hat{n})=\hat{r}, \sum_{\varrho=\alpha+1}^{\infty} n_{\varrho}=N-\beta$ and $0 \leq \beta \leq \min \{\alpha, N\}$. Hence the finite sequence $(\hat{n}, \alpha)$ contains exactly $\beta$ numbers 1 and $\alpha-\beta$ numbers 0 . Because $\beta$ is determined by $\hat{r}$, the notation is a bit redundant, but it turns out to be useful.

Now it is supposed that $\alpha$ and $N$ are fixed numbers. Then, Definition 6.2 yields the following

Consequence 6.3. 1.) The set $\mathcal{N}_{\beta}(\hat{r}), 0 \leq \beta \leq \min \{\alpha, N\}$ is finite, more precisely, card $\mathcal{N}_{\beta}(\hat{r})=\left(\begin{array}{l}\alpha \\ \beta\end{array}\right)$. Therefore the subspace $\mathcal{H}_{-}^{N}(\hat{r})$ of $\mathcal{H}_{-}^{N}$ spanned by the $\Psi_{N}^{-}(\hat{n})$ for $\hat{n} \in \mathcal{N}_{\beta}(\hat{r})$ has dimension $\left(\begin{array}{l}\alpha \\ \beta\end{array}\right)$.

2.) The sets $\mathcal{N}_{\beta}(\hat{r})$ and $\mathcal{N}_{\beta^{\prime}}\left(\hat{r}^{\prime}\right)$ are disjoint if $\hat{r} \neq \hat{r}^{\prime}$. Moreover $\beta=\beta^{\prime}$, if and only if the sequences $\hat{r}, \hat{r}^{\prime}$ contain the same number of elements 1 . Then for each $\hat{n} \in \mathcal{N}_{\beta}(\hat{r})$ there is an $\hat{n}^{\prime} \in \mathcal{N}_{\beta}\left(\hat{r}^{\prime}\right)$ such that $\left(\hat{n}^{\prime}, \alpha\right)=(\hat{n}, \alpha)$.

3.) Let $\mathcal{B}_{N}^{-}$be the $O N B$ of $\mathcal{H}_{-}^{N}$ defined by $(A .1 .13)$, and let $\Psi_{N}^{-}(\hat{n}) \in \mathcal{B}_{N}^{-}$. Then there is exactly one $\beta$ so that $\hat{n} \in \mathcal{N}_{\beta}(\alpha, \hat{n})$. Hence the sets $\mathcal{N}_{\beta}(\hat{r})$ with $\hat{r}=\left(n_{\alpha+1}, n_{\alpha+2}, \ldots\right)$ containing $N-\beta$ numbers 1 and $0 \leq \beta \leq \min \{\alpha, N\}$ form a complete disjoint dissection of the set of all $\hat{n} \in B Z F$ for which $\sum_{\varrho} n_{\varrho}=N$. 
4.) From the above parts 2 and 3 one concludes that the spaces $\mathcal{H}_{-}^{N}(\hat{r})$ are orthogonal for different $\hat{r}$, and that they span $\mathcal{H}_{-}^{N}$, i.e.

$$
\mathcal{H}_{-}^{N}=\bigoplus_{\hat{r}} \mathcal{H}_{-}^{N}(\hat{r})
$$

Later on a restriction of the set $\mathcal{D}$ of all difference sequences (cf. Notation 3.8) is needed.

Definition 6.4. $\mathcal{D}_{\alpha}$ is the set of all $\hat{d} \in \mathcal{D}$, for which $d_{\varrho}=0$ if $\varrho>\alpha$. In addition let $\mathcal{D}_{j \alpha}:=\mathcal{D}_{j} \cap \mathcal{D}_{\alpha}, j=0,1,2$.

Therefore the sets $\mathcal{D}_{j \alpha}$ are again disjoint, and $\mathcal{D}_{0 \alpha}=\mathcal{D}_{0}=\{\hat{o}\}$.

6.1.3: Finally a lemma is proved which is basic for the further considerations.

Proposition 6.5. If $\hat{n} \in \mathcal{N}_{\beta}(\hat{r}), 0 \leq \beta \leq \min \{\alpha, N\}$ and if $\hat{d} \in \mathcal{D}_{\alpha}, \hat{d} \neq \hat{o}$, then either $\hat{n}+\hat{d} \in \mathcal{N}_{\beta}(\hat{r})$ or $\hat{n}+\hat{d} \notin B Z F$.

Proof. The proof is complete if one can show that $\hat{n}+\hat{d} \in \mathcal{N}_{\beta}(\hat{r})$ is equivalent to $\hat{n}+\hat{d} \in B Z F$. First, if $\hat{n}+\hat{d} \in \mathcal{N}_{\beta}(\hat{r})$, then $\hat{n}+\hat{d} \in B Z F$ holds. Second, it follows from $\hat{n}+\hat{d} \in B Z F$, that the (two or one) numbers 1 in $\hat{d}$ must be at positions where there are 0 in $\hat{n}$. Likewise, the (two or one) numbers -1 in $\hat{d}$ must be at positions, where numbers 1 are in $\hat{n}$. Because of $\hat{d} \in \mathcal{D}_{\alpha}$, the sequence $(\hat{n}+\hat{d}, \alpha)$ has the same quantity $\beta$ of numbers 1 as $(\hat{n}, \alpha)$ has, and $\hat{r}:=(\alpha, \hat{n})=(\alpha, \hat{n}+\hat{d})$ because $\hat{d}$ does not affect $\hat{r}$. Thus $\hat{n}+\hat{d} \in \mathcal{N}_{\beta}(\hat{r})$, so that the proof is complete.

\subsection{General properties of the matrix of $\hat{H}_{N}^{-}$}

6.2.1: In what follows the definitions and results of Section 6.1 are applied for the special choice $\alpha=\bar{\alpha}$ with a properly chosen $\bar{\alpha}$. Moreover, the ONBs $\mathcal{B}_{2}^{-}$and $\mathcal{B}_{N}^{-}$are those which are defined via the Hartree-Fock procedure in the Sections 4.2 and 4.3 .

6.2.2: In this subsection the first part of Proposition 6.1 is proved. In order to do so, the matrix representation of $\hat{H}_{N}^{-}$with respect to $\mathcal{B}_{N}^{-}$is used. The proof is complete, if one shows that for $\hat{n} \in \mathcal{N}_{\beta}(\hat{r})$ and $\hat{n}^{\prime} \in \mathcal{N}_{\beta^{\prime}}\left(\hat{r}^{\prime}\right)$ with $\hat{r} \neq \hat{r}^{\prime}:$

$$
\left\langle\hat{n}^{\prime}\left|\hat{H}_{N}^{-}\right| \hat{n}\right\rangle=0 .
$$

There are three possibilities for the pair $\hat{n}^{\prime}, n$. 
If $\hat{n}^{\prime}-\hat{n} \notin \mathcal{D}$, it follows from Formula (4.3) that (6.3) holds.

If $\hat{n}^{\prime}-\hat{n} \in \mathcal{D} \backslash \mathcal{D}_{\bar{\alpha}}$, by Formula (4.4) it is seen that (6.3) holds, too.

If $\hat{n}^{\prime}-\hat{n} \in \mathcal{D}_{\bar{\alpha}}$, then $\hat{n}^{\prime}=\hat{n}+\hat{d} \in B Z F, \hat{d} \in \mathcal{D}_{\bar{\alpha}}$ and $\hat{d} \neq \hat{o}$. Thus it follows from Proposition 6.5 that $\hat{n}^{\prime} \in \mathcal{N}_{\beta}(\hat{r})$. This result contradicts the supposition $\hat{n}^{\prime} \in \mathcal{N}_{\beta^{\prime}}\left(\hat{r}^{\prime}\right)$ and $\hat{r}^{\prime} \neq \hat{r}$. Therefore $\hat{n}^{\prime}-\hat{n} \notin \mathcal{D}_{\bar{\alpha}}$ is true.

Hence (6.3) holds if $\left(\bar{\alpha}, \hat{n}^{\prime}\right) \neq(\bar{\alpha}, \hat{n})$.

If one denotes the restriction of $\hat{H}_{N}^{-}$to the space $\mathcal{H}_{-}^{N}(\hat{r})$ by $\hat{H}_{N}^{-}(\hat{r})$ one obtains

$$
\hat{H}_{N}^{-}=\bigoplus_{\hat{r}} \hat{H}_{N}^{-}(\hat{r})
$$

Thus, part one of Proposition 6.1 has been proved. The proof of the second part is postponed to Section 6.3.

6.2.3: In this subsection therefor some preparatory work will be done. Since the dimension of $\mathcal{H}_{-}^{N}(\hat{r})$ is $\left(\begin{array}{c}\bar{\alpha} \\ \beta\end{array}\right)$ with $0 \leq \beta \leq \min \{\bar{\alpha}, N\}$, it can become gigantic depending on $N, \bar{\alpha}$ and $\beta$. Therefore, aiming at the diagonalization of $\hat{H}_{N}^{-}(\hat{r})$ it is of vital interest to know how many matrix elements $\left\langle\hat{n}^{\prime}\left|\hat{H}_{N}^{-}(\hat{r})\right| \hat{n}\right\rangle$ vanish on principle grounds. This means, how many matrix elements of $\hat{H}_{N}^{-}$ are zero for arbitrary dummy Hamiltonians $\hat{H}_{20}^{-}$respectively their matrices $\hat{E}$. Then in addition further matrix elements of $\hat{H}_{N}^{-}$can be zero for special $\hat{H}_{20}^{-}$. But the last aspect will not be considered in this paper.

Now, from Consequence 4.3 one immediately draws

Conclusion 6.6. Let $\hat{n}^{\prime}, \hat{n} \in \mathcal{N}_{\beta}(\hat{r})$ and let $\hat{n}^{\prime}-\hat{n} \notin \mathcal{D}_{\bar{\alpha}}$, then

$$
\left\langle\hat{n}^{\prime}\left|\hat{H}_{N}^{-}\right| \hat{n}\right\rangle=0 .
$$

Since by Proposition 3.9 the relation

$$
\hat{n}^{\prime}=\hat{n}+\hat{d}_{1}+\cdots+\hat{d}_{L}, \quad \hat{d}_{j} \in \mathcal{D}_{1}
$$

holds, one has a simple criterion to decide whether $\hat{n}^{\prime}-\hat{n} \notin \mathcal{D}_{\bar{\alpha}}$ or not. Especially, if $L>2$ Formula (6.5) is true.

6.2.4: Finally the nondiagonal matrix elements with $\hat{n}^{\prime}-\hat{n}=\hat{d} \in \mathcal{D}_{\bar{\alpha}}, \hat{d} \neq \hat{o}$ are considered. For this purpose let us introduce the following

Notation 6.7. If $\hat{n}^{\prime}, \hat{n} \in B Z F_{N}$. Then $\hat{n}^{\prime}, \hat{n}$ are called $\mathcal{D}_{j \bar{\alpha}}-$ concatenated, $j=0,1,2$, if there is a $\hat{d} \in \mathcal{D}_{j \bar{\alpha}}$ so that $\hat{n}^{\prime}=\hat{n}+\hat{d}$. The $b z f \hat{n}^{\prime}, \hat{n}$ are simply called $\mathcal{D}_{\bar{\alpha}}-$ concatenated if they are $\mathcal{D}_{j \bar{\alpha}}-$ concatenated for $j=0$ or 1 or 2 . 
With the help of this notation we arrive at the

Result 6.8. 1.) For each $\hat{n} \in \mathcal{N}_{\beta}(\hat{r})$, there are exactly

$$
\tau_{1}(\bar{\alpha}, \beta):=\beta(\bar{\alpha}-\beta)
$$

$\mathcal{D}_{1 \bar{\alpha}}$-concatenated $\hat{n}^{\prime} \in \mathcal{N}_{\beta}(r)$. This is because each number 1 out of the $\beta$ numbers 1 in $(\hat{n}, \bar{\alpha})$ can be transposed at each position of the $\bar{\alpha}-\beta$ numbers 0 by a $\hat{d} \in \mathcal{D}_{1 \bar{\alpha}}$.

2.) For each $\hat{n} \in \mathcal{N}_{\beta}(\hat{r})$ there are exactly

$$
\tau_{2}(\bar{\alpha}, \beta):=\left(\begin{array}{l}
\beta \\
2
\end{array}\right)\left(\begin{array}{c}
\bar{\alpha}-\beta \\
2
\end{array}\right)
$$

$\mathcal{D}_{2 \bar{\alpha}}$-concatenated $\hat{n}^{\prime} \in \mathcal{N}_{\beta}(\hat{r})$, where $\left(\frac{\varrho}{2}\right)=0$ if $\varrho=0,1$. This holds because each pair of numbers 1 out of the $\beta$ numbers 1 in $(\hat{n}, \bar{\alpha})$ can be brought at the position of each pair out of the $\bar{\alpha}-\beta$ numbers 0 by a $\hat{d} \in \mathcal{D}_{2 \bar{\alpha}}$.

3.) $\hat{n}^{\prime}, \hat{n} \in \mathcal{N}_{\beta}(\hat{r})$ are $\mathcal{D}_{0 \bar{\alpha}}-$ concatenated exactly if $\hat{n}^{\prime}=\hat{n}$.

4.) For each $\hat{n} \in \mathcal{N}_{\beta}(\hat{r})$ there are exactly

$$
\tau(\bar{\alpha}, \beta):=\tau_{2}(\bar{\alpha}, \beta)+\tau_{1}(\bar{\alpha}, \beta)=\left(\begin{array}{l}
\beta \\
2
\end{array}\right)\left(\begin{array}{c}
\bar{\alpha}-\beta \\
2
\end{array}\right)+\beta(\bar{\alpha}-\beta)
$$

$\mathcal{D}_{\bar{\alpha}}$-concatenated $\hat{n}^{\prime} \neq \hat{n}$.

5.) Finally, let us consider the matrix of $\hat{H}_{N}^{-}(\hat{r})$ with the elements $\left\langle\hat{n}^{\prime}\left|\hat{H}_{N}^{-}\right| \hat{n}\right\rangle, \hat{n}^{\prime}, \hat{n} \in \mathcal{N}_{\beta}(\hat{r})$. Then, in the $\hat{n}^{\prime}$-row there are exactly $\tau(\bar{\alpha}, \beta)$ nondiagonal elements which can be unequal zero, and similarly for $\hat{n}$-columns. Consequently, the number $Z(\bar{\alpha}, \beta)$ of zero nondiagonal elements in each $\hat{n}^{\prime}-$ row or $\hat{n}$-column is

$$
Z(\bar{\alpha}, \beta)=\left(\begin{array}{l}
\bar{\alpha} \\
\beta
\end{array}\right)-\tau(\bar{\alpha}, \beta)-1
$$

According to Conclusion $6.6 Z(\bar{\alpha}, \beta)$ is the number of bzf $\hat{n}$ for which $\hat{n}^{\prime}-\hat{n} \notin \mathcal{D}_{\bar{\alpha}}$ in any $\hat{n}^{\prime}$-row, and likewise for the $\hat{n}$-columns.

\subsection{The matrices of the operators $\hat{H}_{N}^{-}(\hat{r})$}

In this section the second part of Proposition 6.1 will be proved. This runs as follows. 
6.3.1: $\beta=\bar{\alpha} \leq N$. The number $z$ of elements $\hat{n} \in \mathcal{N}_{\bar{\alpha}}(\hat{r})$ is $\left(\begin{array}{l}\bar{\alpha} \\ \bar{\alpha}\end{array}\right)=1$, and the element $\hat{n}$ has the form

$$
\hat{n}=\left(1, \ldots, 1, n_{\bar{\alpha}+1}, \ldots\right) .
$$

Consequently the matrix of $\hat{H}_{N}^{-}(\hat{r})$ is of order one and its element is

$$
\left\langle\hat{n}\left|\hat{H}_{N}^{-}\right| \hat{n}\right\rangle=\hat{\mathcal{E}}(\hat{n}, \hat{o})=\mathcal{E}(\hat{n}, \hat{o}) .
$$

6.3.2: $\beta=\bar{\alpha}-1 \leq N$. 1.) The number $z$ of elements $\hat{n} \in \mathcal{N}_{\bar{\alpha}-1}(\hat{r})$ is $\left(\begin{array}{c}\bar{\alpha} \\ \bar{\alpha}-1\end{array}\right)=$ $\bar{\alpha}$, and the $(\hat{n}, \bar{\alpha})$ for $\hat{n} \in \mathcal{N}_{\bar{\alpha}-1}(\hat{r})$ contain only one 0 and $\bar{\alpha}-1$ numbers 1 .

2.) The $\hat{n} \in N_{\bar{\alpha}-1}(\hat{r})$ are numbered by $\hat{n}=: \hat{n}_{\kappa}$ if 0 is at position $\bar{\alpha}-\kappa$ in $(\hat{n}, \bar{\alpha})$, and $\kappa=0, \ldots, \bar{\alpha}-1$.

3.) Any two elements $\hat{n}^{\prime}, \hat{n} \in \mathcal{N}_{\bar{\alpha}-1}(\hat{r})$ with $\hat{n}^{\prime} \neq \hat{n}$ are $\mathcal{D}_{1 \bar{\alpha}}$-concatenated. This is because the number of $\hat{n} \in \mathcal{N}_{\bar{\alpha}-1}(\hat{r})$, which are $\mathcal{D}_{\bar{\alpha}}$-concatenated with $\hat{n}^{\prime} \neq \hat{n}$, according to $(6.6)$ is

$$
\tau(\bar{\alpha}, \bar{\alpha}-1)=\tau_{1}(\bar{\alpha}, \bar{\alpha}-1)=\bar{\alpha}-1=z-1 .
$$

4.) The matrix of $\hat{H}_{N}^{-}(\hat{r})$ in the present case is a $z \times z=\bar{\alpha} \times \bar{\alpha}$ matrix, which has the elements

$$
\left\langle\kappa^{\prime}\left|\hat{H}_{N}^{-}\right| \kappa\right\rangle:=\left\langle\hat{n}_{\kappa^{\prime}}\left|\hat{H}_{N}^{-}\right| \hat{n}_{\kappa}\right\rangle=\hat{\mathcal{E}}\left(\hat{n}_{\kappa}, \hat{n}_{\kappa^{\prime}}-\hat{n}_{\kappa}\right)
$$

6.3.3: $\beta=\bar{\alpha}-2 \leq N$. 1.) The number $z$ of elements $\hat{n} \in \mathcal{N}_{\bar{\alpha}-2}(\hat{r})$ is $\left(\begin{array}{c}\bar{\alpha} \\ \bar{\alpha}-2\end{array}\right)=$ $\frac{1}{2} \bar{\alpha}(\bar{\alpha}-1)$, and the $(\hat{n}, \bar{\alpha})$ for $\hat{n} \in \mathcal{N}_{\bar{\alpha}-2}(\hat{r})$ contain two numbers 0 and $\bar{\alpha}-2$ numbers 1 .

2.) The $\hat{n} \in \mathcal{N}_{\bar{\alpha}-2}(\hat{r})$ are numbered by $\hat{n}=: \hat{n}_{\kappa \lambda}, \kappa<\lambda$ if the two 0 are at the positions $\bar{\alpha}-\lambda$ and $\bar{\alpha}-\kappa, \quad 0 \leq \kappa<\lambda \leq \bar{\alpha}-1$.

3.) Any two elements $\hat{n}^{\prime}, \hat{n} \in \mathcal{N}_{\bar{\alpha}-2}(\hat{r})$ with $\hat{n}^{\prime} \neq \hat{n}$ are $\mathcal{D}_{\bar{\alpha}}$-concatenated. This is because the number of $\hat{n} \in \mathcal{N}_{\bar{\alpha}-2}(\hat{r})$, which are $\mathcal{D}_{\bar{\alpha}}$-concatenated with $\hat{n}^{\prime} \neq \hat{n}$, according to $(6.6)$ is

$$
\tau(\bar{\alpha}, \bar{\alpha}-2)=\left(\begin{array}{c}
\bar{\alpha} \\
\bar{\alpha}-2
\end{array}\right)-1=z-1 .
$$

4.) The matrix of $\hat{H}_{N}^{-}(\hat{r})$ then is a $z \times z$ matrix with $z=\frac{1}{2} \bar{\alpha}(\bar{\alpha}-1)$, which has the elements

$$
\left\langle\kappa^{\prime}, \lambda^{\prime}\left|\hat{H}_{N}^{-}\right| \kappa, \lambda\right\rangle:=\left\langle\hat{n}_{\kappa^{\prime} \lambda^{\prime}}\left|\hat{H}_{N}^{-}\right| \hat{n}_{\kappa \lambda}\right\rangle=\hat{\mathcal{E}}\left(\hat{n}_{\kappa \lambda}, \hat{n}_{\kappa^{\prime} \lambda^{\prime}}-\hat{n}_{\kappa \lambda}\right) .
$$

6.3.4: $\beta=2<N$. 1.) The number $z$ of elements $\hat{n} \in \mathcal{N}_{2}(\hat{r})$ is $\left(\begin{array}{l}\bar{\alpha} \\ 2\end{array}\right)=\frac{1}{2} \bar{\alpha}(\bar{\alpha}-$ $1)$, and the $(\hat{n}, \bar{\alpha})$ for $\hat{n} \in \mathcal{N}_{2}(\hat{r})$ contain two numbers 1 and $\bar{\alpha}-2$ numbers 0 . 
2.) The $\hat{n} \in \mathcal{N}_{2}(\hat{r})$ are numbered by $\hat{n}=: \hat{n}_{\kappa \lambda}, \kappa<\lambda$, if the two 1 are at positions $\kappa$ and $\lambda, 1 \leq \kappa<\lambda \leq \bar{\alpha}$

3.) Any two $\hat{n}^{\prime}, \hat{n} \in \mathcal{N}_{2}(\hat{r})$ are $\mathcal{D}_{\bar{\alpha}}$-concatenated. This follow via the same argument as in 6.3.3.

4.) Like in 6.3 .3 one obtains the $z \times z$ matrix of $\hat{H}_{N}^{-}(\hat{r})$. It has the elements

$$
\left\langle\kappa^{\prime} \lambda^{\prime}\left|\hat{H}_{N}^{-}\right| \kappa \lambda\right\rangle:=\left\langle\hat{n}_{\kappa^{\prime} \lambda^{\prime}}\left|\hat{H}_{N}^{-}\right| \hat{n}_{\kappa \lambda}\right\rangle=\hat{\mathcal{E}}\left(\hat{n}_{\kappa \lambda}, \hat{n}_{\kappa^{\prime} \lambda^{\prime}}-\hat{n}_{\kappa \lambda}\right) .
$$

6.3.5: $\beta=1, N>2$. 1.) The number $z$ of elements $\hat{n} \in \mathcal{N}_{1}(\hat{r})$ is $\left(\begin{array}{l}\bar{\alpha} \\ 1\end{array}\right)=\bar{\alpha}$, and the $(\hat{n}, \bar{\alpha})$ for $\hat{n} \in \mathcal{N}_{1}(\hat{r})$ contain one number 1 and $\bar{\alpha}-1$ numbers 0 .

2.) The $\hat{n} \in \mathcal{N}_{1}(\hat{r})$ are numbered by $\hat{n}=: \hat{n}_{\kappa}$, if 1 is at position $\kappa, 1 \leq$ $\kappa \leq \bar{\alpha}$.

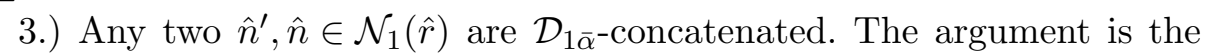
same as in 6.3.2.

4.) Also as in 6.3.2 the matrix $\hat{H}_{N}^{-}(\hat{r})$ is obtained. It is a $z \times z=\bar{\alpha} \times \bar{\alpha}$ matrix having the elements

$$
\left\langle\kappa^{\prime}\left|\hat{H}_{N}^{-}\right| \kappa\right\rangle:=\left\langle\hat{n}_{\kappa^{\prime}}\left|\hat{H}_{N}^{-}\right| \hat{n}_{\kappa}\right\rangle=\hat{\mathcal{E}}\left(\hat{n}_{\kappa}, \hat{n}_{\kappa}^{\prime}-\hat{n}_{\kappa}\right) .
$$

6.3.6: $\beta=0, N>2$. The number $z$ of elements $\hat{n} \in \mathcal{N}_{0}(\hat{r})$ is $\left(\begin{array}{l}\bar{\alpha} \\ 0\end{array}\right)=1$, and the element $\hat{n}$ has the form

$$
\hat{n}=\left(0, \ldots, 0, n_{\bar{\alpha}+1}, \ldots\right) .
$$

Consequently the matrix of $\hat{H}_{N}^{-}(\hat{r})$ is of order 1 and its element is

$$
\left\langle\hat{n}\left|\hat{H}_{N}^{-}\right| \hat{n}\right\rangle=\hat{\mathcal{E}}(\hat{n}, \hat{o})=\mathcal{E}(\hat{n}, \hat{o}) .
$$

6.3.7: $2<\beta<\bar{\alpha}-2, \beta \leq N$. 1.) The number $z$ of elements $\hat{n} \in \mathcal{N}_{\beta}(\hat{r})$ is $\left(\begin{array}{l}\bar{\alpha} \\ \beta\end{array}\right)$. It is larger than the numbers $z$ in the previous cases. Each element $\hat{n} \in \mathcal{N}_{\beta}(\hat{r})$ contains in $(\hat{n}, \alpha)$ at least three numbers 1 and three numbers 0 .

2.) For each $\beta$ not all pairs $\hat{n}^{\prime}, n^{\prime} \in \mathcal{N}_{\beta}(\hat{r})$ are $\mathcal{D}_{\bar{\alpha}}$-concatenated. To prove this proposition it suffices to give an example. Thus, let

$$
\hat{n}^{\prime}=\left(1,1,1, \ldots, 0,0,0, n_{\bar{\alpha}+1}, \ldots\right), \hat{n}=\left(0,0,0, \ldots, 1,1,1, n_{\bar{\alpha}+1}, \ldots\right),
$$

and let $\hat{d}_{j} \in \mathcal{D}, j=1,2,3$ be defined by $d_{j \varrho}=\delta_{j \varrho}-\delta_{\bar{\alpha}+j-3, \varrho}, \varrho \in \mathbb{N}$. Then

$$
\hat{n}^{\prime}=\hat{n}+\hat{d}_{1}+\hat{d}_{2}+\hat{d}_{3}
$$

so that $\hat{n}^{\prime}-\hat{n} \notin \mathcal{D}_{\bar{\alpha}}$. The factual number of non concatinated elements can be calculated from $Z(\bar{\alpha}, \beta)$ as defined by Formula (6.7). 
3.) The elements $\hat{n} \in \mathcal{N}_{\beta}(\hat{r})$ are numbered by $\hat{n}=\hat{n}_{\kappa}, \kappa=1, \ldots, z$ arbitrarily. Then the matrix elements of $\hat{H}_{N}^{-}(\hat{r})$ in the present case are

$$
\left\langle\kappa^{\prime}\left|\hat{H}_{N}^{-}\right| \kappa\right\rangle:=\left\langle\hat{n}_{\kappa^{\prime}}\left|\hat{H}_{N}^{-}\right| \hat{n}_{\kappa}\right\rangle= \begin{cases}\hat{\mathcal{E}}\left(\hat{n}_{\kappa}, \hat{n}_{\kappa^{\prime}}-\hat{n}_{\kappa}\right), & \hat{n}_{\kappa^{\prime}}-\hat{n}_{\kappa} \in \mathcal{D}_{\bar{\alpha}} \\ 0, & \hat{n}_{\kappa^{\prime}}-\hat{n}_{\kappa} \notin \mathcal{D}_{\bar{\alpha}}\end{cases}
$$

6.3.8: Besides the above properties of the matrices of $\hat{H}_{N}^{-}(\hat{r})$ the following result is of practical relevance.

Proposition 6.9. Let the sequences $\hat{r}$ and $\hat{r}^{\prime}$ have the same number $N-\beta$ of elements 1 . If $\hat{n}_{1}, \hat{n}_{2} \in \mathcal{N}_{\beta}(\hat{r})$ and $\hat{n}_{1} \neq \hat{n}_{2}$, there are $\hat{n}_{1}^{\prime}, \hat{n}_{2}^{\prime} \in \mathcal{N}_{\beta}\left(\hat{r}^{\prime}\right), \hat{n}_{1}^{\prime} \neq$ $\hat{n}_{2}^{\prime}$ such that

$$
\left\langle\hat{n}_{1}^{\prime}\left|\hat{H}_{N}^{-}\left(\hat{r}^{\prime}\right)\right| \hat{n}_{2}^{\prime}\right\rangle=\left\langle\hat{n}_{1}\left|\hat{H}_{N}^{-}(\hat{r})\right| \hat{n}_{2}\right\rangle
$$

and vice versa. Thus, the matrices of $\hat{H}_{N}^{-}\left(\hat{r}^{\prime}\right)$ and $\hat{H}_{N}^{-}\left(\hat{r}^{\prime}\right)$ have the same nondiagonal elements.

Proof. For given $\hat{n}_{1}, \hat{n}_{2}$ the bzf $\hat{n}_{1}^{\prime}, \hat{n}_{2}^{\prime}$ are chosen according to Consequence 6.3 as follows: $\left(\hat{n}_{j}^{\prime}, \alpha\right)=\left(\hat{n}_{j}, \alpha\right), j=1,2$. Thus, $\hat{n}_{2}^{\prime}-\hat{n}_{1}^{\prime}=\hat{n}_{2}-\hat{n}_{1}$.

If $\hat{n}_{2}-\hat{n}_{1} \notin \mathcal{D}_{\bar{\alpha}}$, it follows from the proof in Subsection 6.2.2. that Formula (6.21) holds, because both sides are zero.

Now let us assume that $\hat{d}:=\hat{n}_{2}-\hat{n}_{1} \in \mathcal{D}_{\bar{\alpha}}$. Then applying Formula (3.63) yields

$$
\begin{aligned}
\left\langle\hat{n}_{1}\left|\hat{H}_{N}^{-}(\hat{r})\right| \hat{n}_{2}\right\rangle & =\left\langle\hat{n}_{1}\left|\hat{H}_{N}^{-}\right| \hat{n}_{2}\right\rangle \\
& =\sum_{\hat{m}} C\left(\hat{n}_{2}, \hat{m}+\hat{d}, \hat{m}\right) E(\hat{m}+\hat{d}, \hat{m})
\end{aligned}
$$

where the sum runs over all $\hat{m}$ with $\hat{n}_{2}-\hat{m} \in B Z F$ and $\hat{k}:=\hat{m}+\hat{d} \in B Z F_{2}$. Because $\hat{E}(\hat{m}+\hat{d}, \hat{m})=0$, if $k_{\lambda}=1, \lambda>\bar{\alpha}$ or $m_{\kappa}=1, \kappa>\bar{\alpha}$, in Formula (6.22) only such components $k_{\rho}, m_{\sigma}$ are relevant, for which $\rho, \sigma \leq \bar{\alpha}$. Therefore, if the condition $\hat{n}_{2}-\hat{m} \in B Z F$ is satisfied, then also $\hat{n}_{2}^{\prime}-\hat{m} \in B Z F$ holds. The other condition is also satisfied, because $\hat{d}=\hat{n}_{2}^{\prime}-\hat{n}_{1}^{\prime}$. Finally, the inversions, which determine $C(\hat{n}, \hat{m}+\hat{d}, \hat{m})$, only refer to the elements of $\left(\hat{n}_{2}^{\prime}, \alpha\right)=\left(\hat{n}_{2}, \alpha\right)$. Thus one obtains

$$
C(\hat{n}, \hat{m}+\hat{d}, \hat{m})=C\left(\hat{n}^{\prime}, \hat{m}+\hat{d}, \hat{m}\right)
$$

Hence the last term in $(6.22)$ is equal to 


$$
\sum_{\hat{m}} C\left(\hat{n}_{2}^{\prime}, \hat{m}+\hat{d}, \hat{m}\right) E(\hat{m}+\hat{d}, \hat{m})=\left\langle\hat{n}_{1}^{\prime}\left|\hat{H}_{N}^{-}\right| \hat{n}_{2}^{\prime}\right\rangle=\left\langle\hat{n}_{1}^{\prime}\left|\hat{H}_{N}^{-}\left(\hat{r}^{\prime}\right)\right| \hat{n}_{2}^{\prime}\right\rangle .
$$

The proof of the inverse runs the same way.

\subsection{Conclusion}

The decomposition of the matrix of $\hat{H}_{N}^{-}$into orthogonal finite matrices as described in the Sections 6.2 and 6.3 now allows, depending on $N$ and $\bar{\alpha}$, to determine parts of the spectrum of $\hat{H}_{N}^{-}$. Thus, it is only a question of the capacity of the computers available, which parts of the spectrum one can calculate, and it is a question of physical relevance, which parts one wants to calculate. Intuitively, the case $\beta=\bar{\alpha}$ is a Hartree-Fock approximation. This suggests that better approximations are achieved if $\bar{\alpha}$ is greater than $N$, because then the case $\beta=\bar{\alpha}$ cannot occur.

\section{Final Remarks}

\subsection{Summary of the results}

7.1.1: The way of approaching the eigenvalue problem of the Hamiltonian $H_{N}^{-}$presented in the Sections 3 to 6 may at the first glance seem complicated. Therefore it is useful to realize the simple kernel of the procedure. I will present it in the form of a work program which comprises six steps.

$\mathbf{1}^{\text {st }}$ step: As a starting point one formulates the Hamiltonian $\bar{H}_{N}$ to be considered. This is usually done making use of the position-spin representation, i.e. $\bar{H}_{N}$ is an operator in the Hilbert space $\overline{\mathcal{H}}^{N}=\bigotimes^{N}\left(L^{2}\left(\mathbb{R}^{3}\right) \otimes \mathcal{S}^{1}\right)$. The most general form of $\bar{H}_{N}$ for charged particles is due to Breit. It can be found in the literature, e.g. in [14] p. 247.

$2^{\text {nd }}$ step: According to Formula (2.5) one shapes the dummy Hamiltonian $\bar{H}_{20}^{-}:=\bar{H}_{2}^{-}\left(\gamma_{0}\right), \gamma_{0}^{-1}=N-1$, belonging to $\bar{H}_{N}$. Then one determines the orhonormal system $\mathcal{O}_{1}$ via the Hartree-Fock procedure for $\bar{H}_{20}^{-}$(Cf. Appendix A.3.), an equivalent method or an approximation. Eventually, the operators $\bar{H}_{N}^{-}$and $\bar{H}_{20}^{-}$are restricted to the spaces $\mathcal{H}_{-}^{N}, \mathcal{H}_{-}^{2}$ with $\mathcal{H}^{1}=$ span $\mathcal{O}_{1}$ and are denoted $H_{N}^{-}$and $H_{20}^{-}$.

$3^{r d}$ step: In order to determine the matrix $E$ of $H_{20}^{-}$one has to choose an ONB $\mathcal{B}_{1}$ of $\mathcal{H}^{1}$. As explained in Section 4.3, the best choise is $\mathcal{B}_{1}=\mathcal{O}_{1}$. Then, using the ONB $\mathcal{B}_{2}^{-} \subset \mathcal{H}_{-}^{2}$ the matrix elements $E(\hat{k}, \hat{m})$ are calculated (cf. e.g. (A.1.10), (A.1.13)). 
$4^{\text {th }}$ step: From the matrix $E$ one obtains the truncated matrix $\hat{E}$ by replacing the "small" elements $E(\hat{k}, \hat{m}), \hat{k} \neq \hat{m}$ of $E$ by zeros as described in Section 4.4. The matrix $\hat{E}$ depends on a number $\bar{\alpha}$ and determines the operator $\hat{H}_{20}^{-}$.

$5^{\text {th }}$ step: The matrix elements $\hat{\mathcal{E}}(\hat{n}, \hat{d})$ of $\hat{H}_{N}^{-}=\Omega_{N}^{-}\left(\hat{H}_{20}^{-}\right)$are calculated from the matrix elements $\hat{E}(\hat{k}, \hat{m})$ the same way as the $\mathcal{E}(\hat{n}, \hat{d})$ are calculated from $E(\hat{k}, \hat{m})$ in Section 3.5. In this connection the general results of the Sections 6.1 and 6.2 are useful.

$6^{\text {th }}$ step: One determines the orthogonal submatrices of the matrix of $\hat{H}_{N}^{-}$according to Section 6.3 and diagonalizes them as many as possible numerically or analytically.

Then one can try to obtain error estimates applying suitable results of the theory of spectral approximation.

7.1.2: As mentioned at the end of Section 6.4, the lowest energy levels of $\hat{H}_{N}^{-}$ are not simply Hartree-Fock-like approximations of the true values, if $N$ is smaller than $\bar{\alpha}$. Thus in this case the method presented here reproduces also those results, which are obtained by other methods like density functional theory (DFT) or configuration interaction method (CI). Summing up, it is intuitively clear that the approximation of $H_{N}^{-}$by $\hat{H}_{N}^{-}$is the better the smaller the number $N$ and the larger the parameter $\bar{\alpha}$, which in turn is limited by the capacity of computers. My colleague Arno Schindlmayr is preparing an application of the proposed method.

\subsection{Finite procedures}

7.2.1: The program described in Section 7.1.1 is a work program in a strict sense only if all its steps could be carried through in finite time. Thus, the critical points are found in those steps which contain infinite tasks. The first and decisive one is the determination of the infinite ONB $\mathcal{O}_{1}$ in the second step. For one has to expect that in most cases $\mathcal{O}_{1}$ can neither analytically nor numerically be calculated completely. Hence, what can be performed is the determination of a finite part $\mathcal{O}_{f 1}$ of $\mathcal{O}_{1}$, i.e. its elements up to a number $R$.

However, if only $\mathcal{O}_{f 1}$ is available, the method described here does not brake down. Rather the work program formulated in Section 7.1.1 can also be carried through for $\mathcal{O}_{f 1}$ instead of $\mathcal{O}_{1}$. The only question is, which of the obtained results are of physical interest. 
In order to get an answer let us use the following obvious notation:

$$
\mathcal{B}_{f 1}=\mathcal{O}_{f 1}, \mathcal{H}_{f}^{1}, \mathcal{H}_{f-}^{2}, \mathcal{B}_{f 2}^{-}, \mathcal{B}_{f N}^{-}, \mathcal{H}_{f-}^{N}, H_{f 20}^{-}, \hat{H}_{f 20}^{-}, H_{f N}^{-}, \hat{H}_{f N}^{-}, \hat{E}_{f}, \hat{\mathcal{E}}_{f}
$$

Moreover, as already introduced above, $R$ is the number of elements of $\mathcal{B}_{f 1}$.

Now, because the reduced work program depends on the three parameters $N, R$ and $\bar{\alpha}$, the above question can be answered as follows.

1.) If $N>R$, there are no vectors unequal zero in $\mathcal{H}_{f-}^{N}$. Therefore this case has to be excluded.

2.) If $N=R$, the Hilbert space $\mathcal{H}_{f-}^{N}$ is 1-dimensional, so that the $N$ particle Hamiltonian $\hat{H}_{f N}^{-}=H_{f N}^{-}$has one eigenvalue of Hartree-Fock type. This result is of minor interest.

Thus, in order to get better results one needs an $R$ which is "sufficiently" larger than $N$.

3.) Let $\bar{\alpha} \leq N<R$. Then, according to the classification described in Section 6.3, for each $\beta$ satisfying $\max \{0, \bar{\alpha}+N-R\} \leq \beta \leq \bar{\alpha}$ at least one submatrix of the matrix of $\hat{H}_{f N}^{-}$exists, which in principle can be diagonalized numerically.

4.) Let $N<\bar{\alpha}<R$. Then, as in point 3 , for each $\beta$ with $\max \{0, \bar{\alpha}+$ $N-R\} \leq \beta \leq N$ there is again at least one submatrix of the matrix of $\hat{H}_{f N}^{-}$, which in principle can be diagonalized numerically.

5.) If $N<\bar{\alpha}=R$, then $\beta=N$. Therefore, there is only one submatrix of the matrix of $\hat{H}_{f N}^{-}$, which is identical with the matrix of $\hat{H}_{f N}^{-}$. Moreover $\hat{H}_{f N}^{-}=H_{f N}^{-}$. Hence, this case is optimal, but it possibly can not be treated numerically because $R$ is too large.

The result of the above considerations now reads: only the cases 3., 4. and 5. can be of physical interest.

7.2.2: Thus, the question arises, are they. In other words, what can be said about the spectrum of $\hat{H}_{N}^{-}$by studying $\hat{H}_{f N}^{-}$. The answer is given by

Proposition 7.1. The spectrum of $\hat{H}_{f N}^{-}$is contained in the spectrum of $\hat{H}_{N}^{-}$. Thus, the finite work program does not change the eigenvalues of $\hat{H}_{N}^{-}$, rather it delivers only a subset of them.

The proof runs as follows. It suffices to show that the matrices of $\hat{H}_{f N}^{-}$and $\hat{H}_{N}^{-}$with respect to the ONB $\mathcal{B}_{f N}^{-}$are identical.

Let $\mathcal{M}_{f L}$ be the set of all $\hat{l} \in B Z F_{L}$ such that $l_{\varrho}=0$, if $\varrho>R$. Then $\hat{n} \in \mathcal{M}_{f N}$ exactly if $\Psi_{N}^{-}(\hat{n}) \in \mathcal{B}_{f N}^{-}$. Now let $\hat{k}, \hat{m} \in B Z F_{2}$ and $\hat{n}^{\prime}, \hat{n} \in \mathcal{M}_{f N}$. 
If $\hat{n}-\hat{m} \in B Z F$ and $\hat{n}^{\prime}-\hat{k} \in B Z F$, then $\hat{k}, \hat{m} \in \mathcal{M}_{f 2}$. This implies

$$
\hat{E}_{f}(\hat{k}, \hat{m})=\hat{E}(\hat{k}, \hat{m}) .
$$

In addition let $\mathcal{D}_{f}$ be the set of all $\hat{d}:=\hat{k}-\hat{m}, \hat{k}, \hat{m} \in \mathcal{M}_{f 2}$. Thus $d_{\varrho}=0$, if $\varrho>R$, for each $d \in \mathcal{D}_{f}$.

By definition, $\hat{\mathcal{E}}_{f}(\hat{n}, \hat{d})$, where $\hat{n} \in \mathcal{M}_{f N}$ and $\hat{d} \in \mathcal{D}_{f}$, is constructed from $\hat{E}_{f}(\hat{k}, \hat{m})$ via Formula (3.63) like $\hat{\mathcal{E}}(\hat{n}, \hat{d})$ from $\hat{E}(\hat{k}, \hat{m})$ (or $\mathcal{E}(\hat{n}, \hat{d})$ from $E(\hat{k}, \hat{m}))$. Therefore, by Formula (7.1) one obtains

$$
\hat{\mathcal{E}}_{f}(\hat{n}, \hat{d})=\hat{\mathcal{E}}(\hat{n}, \hat{d})
$$

so that by use of (3.61)

$$
\left\langle\hat{n}^{\prime}\left|\hat{H}_{f N}^{-}\right| \hat{n}\right\rangle=\left\langle\hat{n}^{\prime}\left|\hat{H}_{N}^{-}\right| \hat{n}\right\rangle .
$$

Finally, if $\hat{n} \in \mathcal{M}_{f N}$ and $\hat{n} \in \mathcal{N}_{\beta}(\hat{r}), \hat{r}=(\bar{\alpha}, \hat{n})$, then $\mathcal{N}_{\beta}(\hat{r}) \subset \mathcal{M}_{f N}$. Therefore, corresponding submatrices of $\hat{H}_{f N}^{-}$and $\hat{H}_{N}^{-}$have the same shape. Hence, they are identical.

This result guarantees the practical applicability of the finite work program.

\section{Appendix A.}

\section{A.1. Glossary}

A.1.1: The formalism briefly described in this section was mainly developed by Cook [10] and by Schroeck [11]. The purpose of this appendix is fixing notation and formulating some few results, which are used throughout the paper.

The starting point is an axiom of $Q M$ that reads: Let $\mathcal{H}^{1}$ be the Hilbert space of a system containing only one particle of a certain kind. Then the Hilbert space of a system containing $N$ particles of the same kind is the symmetric or the antisymmetric subspace of the $N$-fold tensor product $\mathcal{H}^{N}:=\bigotimes^{N} \mathcal{H}^{1}$. Likewise the Hilbert spaces of systems composed of different kinds of particles are subspaces of appropriate tensor products of one-particle Hilbert spaces.

In what follows the tensor product $\otimes$ of Hilbert spaces is understood to be a complete space. But the noncomplete tensor product of linear manifolds is a noncomplete linear manifold. This product is denoted $\underline{\otimes}$. 
The inner product in $\mathcal{H}^{N}$, denoted $\langle\cdot, \cdot\rangle$ or $\langle\cdot, \cdot\rangle_{N}$, is defined as usual by the inner product $\langle\cdot, \cdot\rangle_{1}$ in $\mathcal{H}^{1}$ in the following way. If $f=f_{1} \otimes \cdots \otimes f_{N}$ and $g=g_{1} \otimes \cdots \otimes g_{N}$, then

$$
\langle f, g\rangle_{N}=\left\langle f_{1}, g_{1}\right\rangle_{1} \cdots\left\langle f_{N}, g_{N}\right\rangle_{1} .
$$

By linear and continuous extension $\langle\cdot, \cdot\rangle_{N}$ is defined on $\mathcal{H}^{N}$.

The tensor structure of the $N$-particle Hilbert spaces implies the following

Proposition A.1.1. Let $\mathcal{B}_{1}:=\left\{\phi_{\lambda}: \lambda \in \mathbb{N}\right\}$ be an orthonormal basis (ONB) in $\mathcal{H}^{1}$. Then

$$
\mathcal{B}_{N}:=\left\{\phi_{\lambda_{1}} \otimes \cdots \otimes \phi_{\lambda_{N}}: \lambda_{j} \in \mathbb{N}, j=1, \ldots, N\right\}
$$

is an $O N B$ in $\mathcal{H}^{N}$.

Throughout this paper the abbreviation is used:

$$
\phi_{\lambda_{1} \cdots \lambda_{N}}=\phi_{\lambda_{1}} \otimes \cdots \otimes \phi_{\lambda_{N}} .
$$

A.1.2: Let $\mathcal{S}_{N}$ be the symmetric group, and let $P \in \mathcal{S}_{N}$. Then the operator $U(P)$ of the exchange of particles is defined by

$$
U(P) \phi_{\kappa_{1} \cdots \kappa_{N}}=\phi_{\kappa_{P^{-1}(1)} \cdots \kappa_{P^{-1}(N)}} .
$$

and by continuous linear extension.

The operator $U(P)$ has the following properties.

Proposition A.1.2. 1.) $U(P)$ is invariant under a change of the $O N B$.

2.) $U(P)$ is defined on $\mathcal{H}^{N}$ and is unitary, i.e. $U(P) U^{\star}(P)=1$. Moreover

$$
U^{\star}(P)=U\left(P^{-1}\right), \quad U(P Q)=U(P) U(Q) .
$$

With the help of $U(P), P \in \mathcal{S}_{N}$ the symmetrizer and the antisymmetrizer are defined by

$$
S_{N}^{ \pm}=\frac{1}{N !} \sum_{P \in \mathcal{S}_{N}} \sigma^{ \pm}(P) U(P)
$$

with $\sigma^{+}(P)=1$ and $\sigma^{-}(P)=(-1)^{J(P)}$, where $J(P)$ is either the number of inversions of $P$ or equivalently the number of transpositions forming $P$. 
Some useful properties of the operators $S_{N}^{ \pm}$are summarized in the next Proposition A.1.3. $S_{N}^{ \pm}$are projections defined on $\mathcal{H}^{N}$. Moreover

$$
U(P) S_{N}^{ \pm}=S_{N}^{ \pm} U(P)=\sigma^{ \pm}(P) S_{N}^{ \pm},
$$

$$
S_{M+K}^{ \pm}\left(S_{M}^{ \pm} \phi_{\mu_{1} \cdots \mu_{M}} \otimes S_{K}^{ \pm} \phi_{\kappa_{1} \cdots \kappa_{K}}\right)=S_{M+K}^{ \pm}\left(\phi_{\mu_{1} \cdots \mu_{M}} \otimes \phi_{\kappa_{1} \cdots \kappa_{K}}\right) .
$$

Then the physically relevant subspaces of $\mathcal{H}^{N}$ are $\mathcal{H}_{ \pm}^{N}=S_{N}^{ \pm}\left[\mathcal{H}^{N}\right]$ where + stands for bosons and - for fermions. Thus

$$
\mathcal{H}^{N}=\mathcal{H}_{+}^{N} \oplus \mathcal{H}_{-}^{N} \oplus \mathcal{H}_{r}^{N}
$$

where $\oplus$ is the orthogonal sum as usual.

A special role in this paper play some orthonormal bases of $\mathcal{H}_{ \pm}^{N}$, which are defined by

Proposition A.1.4. 1.) The set $\mathcal{B}_{N}^{+}$of all vectors

$$
\Psi_{\kappa_{1} \cdots \kappa_{N}}^{+}:=\frac{\sqrt{N !}}{\sqrt{\Pi_{j} n_{\kappa_{j}} !}} S_{N}^{+} \phi_{\kappa_{1} \cdots \kappa_{N}}
$$

with $\kappa_{1} \leq \cdots \leq \kappa_{N}$ and $n_{\kappa_{j}}=\sum_{\alpha=1}^{N} \delta_{\kappa_{j} \kappa_{\alpha}}$ is an ONB in $\mathcal{H}_{+}^{N}$. Moreover $\sum_{j} n_{\kappa_{j}}=N$.

2.) The set $\mathcal{B}_{N}^{-}$of all vectors

$$
\Psi_{\kappa_{1} \cdots \kappa_{N}}^{-}:=\sqrt{N !} S_{N}^{-} \phi_{\kappa_{1} \cdots \kappa_{N}}
$$

with $\kappa_{1}<\cdots<\kappa_{N}$ is an $O N B$ in $\mathcal{H}_{-}^{N}$.

A.1.3: For the problems to be treated in this paper notation (A.1.9) and (A.1.10) is not optimal, it can be improved by introducing the sequences of occupation numbers by the following

Definition A.1.5. 1.) Let be given a sequence of indices $\kappa_{1}, \ldots, \kappa_{N}$ as in (A.1.9) or in (A.1.10), where $\kappa_{j} \in \mathbb{N}, j=1, \ldots, N$. Then define the occupation number of $\kappa \in \mathbb{N}$ by

$$
n_{\kappa}=\sum_{j=1}^{N} \delta_{\kappa \kappa_{j}}
$$


and the sequence of all $n_{\kappa}, \kappa \in \mathbb{N}$, abbreviated $b z f$, by

$$
\left(n_{1}, n_{2}, \ldots\right)=: \hat{n}
$$

2.) Moreover let us denote the set of all $b z f$, for + or for - , by $B Z F$. Then the proposition " $\hat{n}$ is a sequence of occupation numbers" is abbreviated by " $\hat{n}$ is a $b z f$ " or by " $\hat{n} \in B Z F$ ". Sometimes it is useful to write $B Z F_{L}$ for the set of all $b z f \hat{l}$ with $\sum_{\varrho} l_{\varrho}=L$.

Consequence A.1.6. 1.) Each sequence of indices $\kappa_{1}, \ldots, \kappa_{N}$ determines uniquely a bzf, and vice versa.

2.) The elements of the $O N B \mathcal{B}_{N}^{ \pm}$can be written this way:

$$
\Psi_{\kappa_{1} \cdots \kappa_{N}}^{ \pm}=: \Psi_{N}^{ \pm}(\hat{n})
$$

This notation turns out to be very useful.

A.1.4: In the next step the question is to be answered which are the physically relevant operators, i.e. the relevant observables in $\mathcal{H}^{N}$. Obviously only those are relevant which leave the spaces $\mathcal{H}_{+}^{N}$ invariant.

Therefore we define: Let $\mathcal{D}_{A} \subset \mathcal{H}^{N}$ be the domain of a selfadjoint operator $A$. Then, if for each $f \in \mathcal{D}_{A} \cap \mathcal{H}_{ \pm}^{N}$ the relation $A f \in \mathcal{H}_{ \pm}^{N}$ holds, the operator $A$ is called a physically relevant observable.

Consequence A.1.7. 1.) A is physically relevant, exactly if

$$
\text { (A.1.14) } \quad A=S_{N}^{+} A S_{N}^{+}+S_{N}^{-} A S_{N}^{-}+S_{N}^{r} A S_{N}^{r} \quad \text { with } \quad S^{r}=1-S_{N}^{+}-S_{N}^{-} \text {. }
$$

2.) $A$ is physically relevant, if for each $P \in \mathcal{S}_{N}$

$$
A=U(P) A U^{\star}(P)
$$

holds, i.e. if $A$ is invariant under permutations of particles.

A.1.5: Many relevant physical observables are defined using tensor products of operators in Hilbert spaces. In order to avoid unnecessary complications here only the tensor product of two operators is introduced, because the extension to more than two factors is straightforward.

Thus let be given two Hilbert spaces $\mathcal{H}_{1}$ and $\mathcal{H}_{2}$ and two densely defined closed operators $A_{1}$ and $A_{2}$. Then $A_{1}^{\star}$ and $A_{2}^{\star}$ exist having domains $\mathcal{D}_{A_{1}^{\star}}$ and $\mathcal{D}_{A_{2}^{\star}}$. 
Now form the (noncomplete) tensor product $\mathcal{D}_{0}:=\mathcal{D}_{A_{1}^{\star}} \otimes \mathcal{D}_{A_{2}^{\star}} \subset \mathcal{H}_{1} \otimes$ $\mathcal{H}_{2}$. It is dense in $\mathcal{H}_{1} \otimes \mathcal{H}_{2}$ and contains only finite linear combinations of the form

$$
f=\sum a_{j} \varphi_{1}^{j} \otimes \varphi_{2}^{j}, \quad \varphi_{\kappa}^{j} \in \mathcal{D}_{A_{\kappa}}, \kappa=1,2
$$

Then the operator $T_{0}\left(A_{1}^{\star}, A_{2}^{\star}\right)$ is defined by

$$
T_{0}\left(A_{1}^{\star}, A_{2}^{\star}\right) f=\sum a_{j}\left(A_{1}^{\star} \varphi_{1}^{j}\right) \otimes\left(A_{2}^{\star} \varphi_{2}^{j}\right) .
$$

Finally the tensor product of $A_{1}$ and $A_{2}$ is defined by

$$
A_{1} \otimes A_{2}=T_{0}\left(A_{1}^{\star}, A_{2}^{\star}\right)^{\star}
$$

Hence, for selfadjoint operators definition (A.1.18) reads

$$
A_{1} \otimes A_{2}=T_{0}\left(A_{1}, A_{2}\right)^{\star}
$$

A very useful tool is given by

Proposition A.1.8. For bounded operators $A_{1}, A_{2}$ the above definition of $A_{1} \otimes A_{2}$ is equivalent to

$$
\left(A_{1} \otimes A_{2}\right) g=\sum_{\lambda \kappa}^{\infty} a_{\lambda \kappa}\left(A_{1} \phi_{\lambda}^{1}\right) \otimes\left(A_{2} \phi_{\kappa}^{2}\right)
$$

where $\left\{\phi_{\lambda}^{\varrho}: \lambda \in \mathbb{N}\right\}$ is an $O N B$ in $\mathcal{H}_{\varrho}, \varrho=1,2$ and $g=\sum_{\lambda \kappa}^{\infty} a_{\lambda \kappa} \phi_{\lambda}^{1} \otimes \phi_{\kappa}^{2} \in$ $\mathcal{H}_{1} \otimes \mathcal{H}_{2}$.

A.1.6: The results of the last section now are applied to transfer observables of $M$-particle systems into observables of $N$-particle systems, $M<N$. Some basic results in this connection are contained in the following

Proposition A.1.9. 1.) Let $A_{M}$ be selfadjoint in $\mathcal{H}^{M}$ and let 1 be the identity operator in $\mathcal{H}^{N-M}$. Then

$$
\left(A_{M} \otimes 1\right)^{\star}=A_{M} \otimes 1
$$

2.) If $A_{M}$ is bounded, then

$$
\left\|A_{M} \otimes 1\right\|=\left\|A_{M}\right\| .
$$


3.) If $\left(A_{M}+B_{M}\right)^{\star}=A_{M}^{\star}+B_{M}^{\star}$, then

$$
\left(A_{M}+B_{M}\right) \otimes 1 \supset\left(A_{M} \otimes 1\right)+\left(B_{M} \otimes 1\right)
$$

The =-sign holds if the domains of both sides are equal, which is the case if $A_{M}, B_{M}$ are bounded and have domain $\mathcal{H}^{M}$.

4.) If $A_{M}$ or $B_{M}$ is bounded then

$$
\left(A_{m} \otimes 1\right)\left(B_{M} \otimes 1\right)=\left(A_{M} B_{M} \otimes 1\right)
$$

5.) From (A.1.7) one concludes that

$$
S_{N}^{ \pm}\left(S_{M}^{ \pm} \otimes 1\right)=S_{N}^{ \pm}=\left(S_{M}^{ \pm} \otimes 1\right) S_{N}^{ \pm}
$$

A.1.7: The operator which defines the physically relevant transfer from $\mathcal{H}^{M}$ to $\mathcal{H}^{N}, M<N$ is given by

(A.1.25) $\Omega_{N}\left(A_{M}\right):=$

$$
(M !(N-M) !)^{-1} \sum_{P \in \mathcal{S}_{N}} U(P)\left(A_{M} \otimes 1 \otimes \cdots \otimes 1\right) U^{\star}(P),
$$

where $A_{M}$ is a densely defined closed linear operator in $\mathcal{H}^{M}$ and where 1 is the identity operator on $\mathcal{H}^{1}$. It has the following properties.

Proposition A.1.10. 1.) The equation

$$
U(Q) \Omega_{N}\left(A_{M}\right) U^{\star}(Q)=\Omega_{N}\left(A_{M}\right)
$$

holds for each $Q \in \mathcal{S}_{N}$ so that $\Omega_{N}\left(A_{M}\right)$ is indeed physically relevant if it is selfadjoint.

2.) If $A_{M}$ is bounded and selfadjoint with domain $\mathcal{H}^{M}$ the operator $\Omega_{N}\left(A_{M}\right)$ is bounded and selfadjoint with domain $\mathcal{H}^{N}$.

3.) If $A_{M}$ is unbounded and selfadjoint then $\Omega_{N}\left(A_{M}\right)$ is not necessarily selfadjoint. But it is symmetric if it is densely defined.

A.1.8: Though the operators $\Omega_{N}\left(A_{M}\right)$ are physically relevant, they are not of interest in a strict sense, if one wants to consider only systems with one kind of particles as is the case in this paper. Then only the spaces $\mathcal{H}_{ \pm}^{N} \subset$ $\mathcal{H}^{N}$ are of interest and the operators defined therein. Thus the following 
definition is natural for the operators of proper physical relevance:

$$
\Omega_{N}^{ \pm}\left(A_{M}\right)=S_{N}^{ \pm} \Omega_{N}\left(A_{M}\right) S_{N}^{ \pm} .
$$

As in the preceding sections here $\Omega_{N}^{ \pm}\left(A_{M}\right)$ is studied only for selfadjoint $A_{M}$. But the definition itself is much more general. The operators $\Omega_{N}^{ \pm}\left(A_{M}\right)$ have some properties, which are of special interest in this paper.

Proposition A.1.11. 1.) For each $P \in \mathcal{S}_{N}$ the following relations hold:

$$
\begin{aligned}
\Omega_{N}^{ \pm}\left(A_{M}\right) & =\left(\begin{array}{c}
N \\
M
\end{array}\right) S_{N}^{ \pm}\left(A_{M} \otimes 1 \cdots \otimes 1\right) S_{N}^{ \pm} \\
& =\left(\begin{array}{c}
N \\
M
\end{array}\right) S_{N}^{ \pm} U(P)\left(A_{M} \otimes 1 \otimes \cdots \otimes 1\right) U^{\star}(P) S_{N}^{ \pm} .
\end{aligned}
$$

2.) $\Omega_{N}^{ \pm}\left(A_{M}\right)$ is selfadjoint, because $A_{M}$ is selfadjoint by supposition and because $S_{N}^{ \pm}$are projections, i.e. are bounded.

3.) If $A_{M}$ is bounded, then $\Omega_{N}^{ \pm}\left(A_{M}\right)$ is bounded and

$$
\left\|\Omega_{N}^{ \pm}\left(A_{M}\right)\right\| \leq\left(\begin{array}{c}
N \\
M
\end{array}\right)\left\|A_{M}\right\| .
$$

4.) If $A_{M}+B_{M}, A_{M}$ and $B_{M}$ are selfadjoint, Formula (A.1.22) implies the relation

$$
\Omega_{M}^{ \pm}\left(A_{M}+B_{M}\right) \supset \Omega_{N}^{ \pm}\left(A_{M}\right)+\Omega_{N}^{ \pm}\left(B_{M}\right) .
$$

If $A_{M}, B_{M}$ are bounded with domain $\mathcal{H}^{M}$, the $=$ sign holds.

5.) Let $A_{M}+B_{M}$ and $\Omega_{N}^{ \pm}\left(A_{M}\right)+\Omega^{ \pm}\left(B_{M}\right)$ be selfadjoint. Then (A.1.30) is an equation, because a selfadjoint operator cannot have a selfadjoint extension.

6.)Finally, from (A.1.24) and (A.1.28) one concludes that

$$
\Omega_{N}^{ \pm}\left(\Omega_{M}^{ \pm}\left(A_{M}\right)\right)=\Omega_{N}^{ \pm}\left(A_{M}\right)
$$

\section{A.2. Dummy Hamiltonians}

A.2.1: In order to formulate explicitly the dummy Hamiltonians for electronic systems it is advisably to work with representations of Hilbert spaces instead of the abstract versions used elsewhere in this paper. For our purposes the position-spin representation is most useful. Therefore the Hilbert 
spaces we are working with are

(A.2.1) $\mathcal{H}_{-}^{N} \subset \overline{\mathcal{H}}^{N}=\bigotimes^{N}\left(L^{2}\left(\mathbb{R}^{3}\right) \otimes \mathcal{S}^{1}\right), \quad \mathcal{H}_{-}^{2} \subset \overline{\mathcal{H}}^{2}=\bigotimes^{2}\left(L^{2}\left(\mathbb{R}^{3}\right) \otimes \mathcal{S}^{1}\right)$,

where $\mathcal{S}^{1}$ is the complex vector space of spin functions $u:\{1,-1\} \rightarrow \mathbb{C}$ which is spanned by the ONB $\left\{\delta_{1, s}, \delta_{-1, s}\right\}$.(Cf. also Subsection 3.1.1.) This choice fits into the abstract formulations by the following definition of the tensor product. Let $x \in \mathbb{R}, s \in\{1,-1\}$, and $z:=(x, s)$. Then, if $f_{1}, f_{2} \in \overline{\mathcal{H}}^{1}=$ $L^{2}\left(\mathbb{R}^{3}\right) \otimes \mathcal{S}^{1}$, one defines $f_{1} \otimes f_{2}$ by

$$
\left(f_{1} \otimes f_{2}\right)\left(z_{1}, z_{2}\right)=f_{1}\left(z_{1}\right) f\left(z_{2}\right) .
$$

For the sake of simplicity let us assume that with the following two examples the external fields and the interactions are electrostatic. This means that all influences of magnetism and spin are disregarded.

A.2.2: On the above assumptions the Hamiltonian for the $N$ electrons in an atom reads

$$
H_{N}^{-} \supset \frac{1}{2 m_{0}} \sum_{j=1}^{N} P_{j}^{2}-N e_{0}^{2} \sum_{j=1}^{N} \frac{1}{r_{j}}+\frac{1}{2} e_{0}^{2} \sum_{j \neq k}^{N} \frac{1}{r_{j k}},
$$

where $r_{j}=\left|x_{j}\right|$ and $r_{j k}=\left|x_{j}-x_{\kappa}\right|$. The domain of $H_{N}^{-}$is $\mathcal{H}_{-}^{N}$ as defined in Section 3.1.1. Consequently, the atomic dummy Hamiltonian describes "dummy helium" and is defined on $\mathcal{H}_{-}^{2}$. It reads explicitly

$$
H_{20}^{-} \supset \frac{\gamma_{0}}{2 m_{0}}\left(P_{1}^{2}+P_{2}^{2}\right)-2 \gamma_{0} e_{0}^{2}\left(\frac{1}{r_{1}}+\frac{1}{r_{2}}\right)+e_{0}^{2} \frac{1}{r_{12}}
$$

with $\gamma_{0}=(N-1)^{-1}$.

A.2.3: Now let us consider $N$ electrons in a finite lattice, the points of which are given by $y_{\alpha}, \alpha=1, \ldots, N$ each carrying the charge $e_{0}$. Then the Hamiltonian is defined by

$$
H_{N}^{-} \supset \frac{1}{2 m_{0}} \sum_{j=1}^{N} P_{j}^{2}-e_{0}^{2} \sum_{j=1}^{N} \sum_{\alpha=1}^{N} \frac{1}{r_{j \alpha}^{\prime}}+\frac{1}{2} \sum_{j \neq \kappa}^{N} \frac{1}{r_{j \kappa}},
$$

where $r_{j \alpha}^{\prime}=\left|x_{j}-y_{\alpha}\right|$. Thus the dummy solid has a Hamiltonian given by

$$
H_{20}^{-} \supset \frac{\gamma_{0}}{2 m_{0}}\left(P_{1}^{2}+P_{2}^{2}\right)-\gamma_{0} e_{0}^{2} \sum_{\alpha=1}^{N}\left(\frac{1}{r_{1 \alpha}^{\prime}}+\frac{1}{r_{2 \alpha}^{\prime}}\right)+e_{0}^{2} \frac{1}{r_{12}}
$$

and is defined on $\mathcal{H}_{-}^{2}$. 


\section{A.3. Hartree-Fock Procedure}

A.3.1: In what follows a system of two Fermions is considered. For this purpose it is useful to introduce some notation.

1.) Let $\overline{\mathcal{H}}^{1}=L^{2}\left(\mathbb{R}^{3}\right) \otimes S^{1}$, where $S^{1}$ is the space of spin functions spaned by the $O N B\left\{\delta_{1 S}, \delta_{-1 S}\right\}$. Thus the general two-particle space is $\overline{\mathcal{H}}^{2}=\overline{\mathcal{H}}^{1} \otimes$ $\overline{\mathcal{H}}^{1}$ and the space for Fermions is $\overline{\mathcal{H}}_{-}^{2}$.

2.) It is assumed that the Hamiltonian of the system has the form

$$
H_{2}=K \otimes 1+1 \otimes K+W
$$

where $W$ is a multiplication operator densely defined in $\overline{\mathcal{H}}_{-}^{2}$ by a real function $V\left(x, s, x^{\prime}, s^{\prime}\right), x, x^{\prime} \in \mathbb{R}^{3}$ and $s, s^{\prime} \in\{1,-1\}$. The operator $K$ contains the kinetic energy and the external fields. The dummy Hamiltonian is an example of the operators considered here.

3.) The inner product in $\overline{\mathcal{H}}^{1}$ is defined as usual by

$$
\langle f, g\rangle=\sum_{s=-1}^{1} \int \bar{f}(x, s) g(x, s) d x
$$

for $f, g \in \overline{\mathcal{H}}^{1}$.

4.) Some special forms of the inner product appear in the context of Hartree-Fork procedure. Let $\Psi \in \overline{\mathcal{H}}^{2}$ and $f, g \in \overline{\mathcal{H}}^{1}$. Then

$$
\langle g, W \Psi\rangle_{1}(x, s)=\sum_{s^{\prime}} \int \bar{g}\left(x^{\prime}, s^{\prime}\right) V\left(x^{\prime}, s^{\prime}, x, s\right) \Psi\left(x^{\prime}, s^{\prime}, x, s\right) d x
$$

and

$$
\langle g, W f\rangle_{1}(x, s)=\sum_{s^{\prime}} \int \bar{g}\left(x^{\prime}, s^{\prime}\right) V\left(x^{\prime}, s^{\prime}, x, s\right) f\left(x^{\prime}, s^{\prime}\right) d x^{\prime} .
$$

Therefore one obtains

$$
\langle g, W(f \otimes h)\rangle_{1}=\langle g, W f\rangle_{1} h .
$$

5.) From (A.3.4) one concludes that

$$
\langle g, W f\rangle_{1}=\langle W g, f\rangle_{1} .
$$

If $g=f$, the term $\langle f, W f\rangle_{1}$ is the action of the "charge density" $|f|^{2}$ on one particle. Moreover, if the function $V$ is bounded, the term $\langle g, W f\rangle_{1}$ is 
defined for all $g, f \in \overline{\mathcal{H}}^{1}$. For realistic Hamiltonians $\bar{H}_{2}$ of the form (A.3.1) the function $V$ is symmetric, i.e. $V\left(x, s, x^{\prime}, s^{\prime}\right)=V\left(x^{\prime}, s^{\prime}, x, s\right)$.

A.3.2: An essential part of the Hartree-Fock procedure is given by the following

Definition A.3.1. The linear operator

$$
F(\chi)=K+\langle\chi, W \chi\rangle_{1}-\langle\chi, W \cdot\rangle_{1} \chi
$$

is defined for all $\chi \in \overline{\mathcal{H}}^{1}$, for which the domain of $F(\chi)$ is dense in $\overline{\mathcal{H}}^{1} \ominus$ $\operatorname{span}\{\chi\}=: \overline{\mathcal{H}}_{\chi}^{1}$. It is called the Fock operator belonging to $\chi$.

Then for each $\chi$, for which $F(\chi)$ is defined, the following result holds.

Proposition A.3.2. $F(\chi)$ is symmetric in $\overline{\mathcal{H}}_{\chi}^{1}$.

Proof. Let $f, g$ be elements of the domain of $F(\chi)$. Then

$$
\begin{aligned}
\langle f, F(\chi) g\rangle & =\langle f, K g\rangle+\langle\chi \otimes f, W \chi \otimes g\rangle-\langle\chi \otimes f, W g \otimes \chi\rangle \\
& =\langle K f, g\rangle+\langle W \chi \otimes f, \chi \otimes g\rangle-\langle W \chi \otimes f, g \otimes \chi\rangle \\
& =\langle F(\chi) f, g\rangle .
\end{aligned}
$$

A.3.3: Now the Hartree-Fock procedure can be described by the following two steps.

$\mathbf{1}^{\text {st }}$ step: Determine two elements $\phi_{1}, \phi_{2}$ of $\overline{\mathcal{H}}^{1}$ and two real numbers $e_{12}$ and $e_{21}$ such that the equations

$$
\begin{aligned}
& F\left(\phi_{2}\right) \phi_{1}=e_{21} \phi_{1} \\
& F\left(\phi_{1}\right) \phi_{2}=e_{12} \phi_{2}
\end{aligned}
$$

are satisfied. In addition let $e_{12} \leq e_{21}$.

$\mathbf{2}^{\text {nd }}$ step: Determine normed elements $\phi_{\kappa} \in \overline{\mathcal{H}}_{\phi_{1}}^{1}, \kappa=3,4, \ldots$ and real numbers $e_{1 \kappa}$ such that the equations

$$
F\left(\phi_{1}\right) \phi_{\kappa}=e_{1 \kappa} \phi_{\kappa}
$$

hold.

The Hartree-Fock procedure is usually derived via the Ritz variational principle. But this derivation is not of interest in the present context, rather the following consequence. 
Proposition A.3.3. The set $\mathcal{O}_{1}$ of vectors $\phi_{\kappa} \in \mathcal{H}^{1}, \kappa=1,2,3, \ldots$ obtained from (A.3.8) and (A.3.9) is an orthonormal system in $\overline{\mathcal{H}}^{1}$.

Proof. From the definition of the Fock operator and from the Formulae (A.3.8), (A.3.9) it follows that $\phi_{\kappa} \in \overline{\mathcal{H}}_{\phi_{1}}^{1}, \kappa \geq 2$. Hence $\left\langle\phi_{1}, \phi_{\kappa}\right\rangle=0$ for all $\kappa \geq 2$. Since the Fock operator $F\left(\phi_{1}\right)$ is a symmetric linear operator in $\overline{\mathcal{H}}_{\phi_{1}}^{1}$, all its eigenspaces are orthogonal for different eigenvalues. Therefore the set of eigenvectors $\phi_{\kappa}, \kappa \geq 2$ can be chosen such that it is an orthonormal system in $\overline{\mathcal{H}}_{\phi_{1}}^{1}$. Hence, $\mathcal{O}_{1}$ is an orthonormal system in $\overline{\mathcal{H}}^{1}$.

A.3.4: The Hartree-Fock procedure is not only a method determining the set $\mathcal{O}_{1}$, rather it is also used for an approximate diagonalization of $\bar{H}_{2}$. This runs as follows. Let

$$
\Psi_{\kappa \lambda}^{-}=\frac{1}{\sqrt{2}}\left(\phi_{\kappa} \otimes \phi_{\lambda}-\phi_{\lambda} \otimes \phi_{\kappa}\right)
$$

for $\kappa<\lambda$ and let

$$
E_{\kappa \lambda}=\left\langle\Psi_{\kappa \lambda}^{-}, H_{2} \Psi_{\kappa \lambda}^{-}\right\rangle
$$

Then the Hartree-Fock approximation of $\bar{H}_{2}$ is given by the diagonal operator

$$
\hat{H}_{2}^{-}=\sum_{\kappa \lambda} E_{\kappa \lambda} \Psi_{\kappa \lambda}^{-}\left\langle\Psi_{\kappa \chi}^{-}, \cdot\right\rangle .
$$

Because normally the discret spectrum of $\bar{H}_{2}$ is bounded, the operator $\hat{H}_{2}^{-}$ is bounded too. $\hat{H}_{2}^{-}$is the best approximation of $\bar{H}_{2}$ using only the Ritz variational principle for vectors of the shape (A.3.10).

By Proposition 4.1 a Hartree-Fock like diagonalization of an $N$-particle Hamiltonian is obtained by Hartree-Fock diagonalizing its dummy Hamiltonian.

\section{Acknowledgment}

I want to thank my colleagues Arno Schindlmayr, Uwe Gerstmann, Thierry Jecko and Jörg Meyer for valuable discussions and critical remarks, and Mr. Wolfgang Rothfritz for correcting my English. 


\section{References}

[1] L. H. Thomas, The calculation of atomic fields. Proc. Cambridge Phil. Soc. 23, 542 (1927).

[2] E. Fermi, A statistical method for the determination of some properties of atoms. Z. Phys. 48, 73 (1928).

[3] D. R. Hartree, The wave mechanics of an atom with a non-coulomb central field. Theory and methods. Proc. Cambridge Phil. Soc. 24, 89 (1928).

[4] V. Fock, Näherungsmethode zur Lösung des quantenmechanischen Mehrkörperproblems. Z. Phys. 61, 126 (1930).

[5] P. Hohenberger and W. Kohn, Inhomogeneous elektron gas. Phys. Rev. 136 B, 864 (1964).

[6] W. Kohn and L. Sham, Selfconsistent equations including exchange and correlation effects. Phys. Rev. 14 A, 1133 (1964).

[7] J. Kohannoff, Electronic Structure Calculations for Solids and Molecules. Cambridge University Press, Cambridge, New York, 2006.

[8] Ohno, K., Esfarjani, K., Kawazoe, Y.: Computational Material Science. Springer Verlag, Berlin, Heidelberg, New York, 1999.

[9] H. Haken, Quantenfeldtheorie des Festkörpers, Teubner Verlag, Stuttgart, 1993.

[10] J. M. Cook, The Mathematics of Second Quantization. Trans. Math. Soc. Vol. 74, 222 (1953).

[11] F. E. Schroeck Jr., Generalization of the Cook Formalism for Fock Space. J. Math. Phys., Vol. 12, 1849 (1971).

[12] F. Chatelin, Spectral Approximation of Linear Oparators. Academic Press, New York, London, Paris, 2011.

[13] T. Kato, Perturbation theory for linear operators. Springer-Verlag, Berlin, Heidelberg, New York, 1966.

[14] G. Ludwig, Die Grundlagen der Quantenmechanik. Springer-Verlag, Berlin, Göttingen, Heidelberg, 1954. 
Department of Physics, University of PAderborn 33098 Paderborn, Germany

E-mail address: j@schroe.de 